\title{
A high-resolution study of isotopic composition and chemical abundances of blue horizontal branch stars in the globular clusters NGC 6397 and NGC 6752^
}

\author{
S. Hubrigg ${ }^{1,2}$, F. Castelli ${ }^{3}$, G. De Silva ${ }^{4}$, J. F. González ${ }^{5}$, Y. Momany ${ }^{1}$, M. Netopil ${ }^{6}$, and S. Moehler ${ }^{4}$ \\ 1 Astrophysical Institute Potsdam, An der Sternwarte 16, 14482 Potsdam, Germany \\ e-mail: shubrig@aip.de \\ 2 European Southern Observatory, Casilla 19001, Santiago 19, Chile \\ 3 Istituto Nazionale di Astrofisica - Osservatorio Astronomico di Trieste, via Tiepolo 11, 34131 Trieste, Italy \\ ${ }^{4}$ European Southern Observatory, Karl-Schwarzschild-Str. 2, 85748 Garching, Germany \\ 5 Complejo Astronómico El Leoncito, Casilla 467, 5400 San Juan, Argentina \\ ${ }^{6}$ Institut für Astronomie der Universität Wien, Türkenschanzstr. 17, 1180 Wien, Austria
}

Received 25 January 2009 / Accepted 18 March 2009

\section{ABSTRACT}

\begin{abstract}
Aims. Large abundance anomalies have been previously detected in Horizontal Branch B-type stars. It has been suggested that at $T_{\text {eff }}>11000 \mathrm{~K}$ the stellar atmospheres become susceptible to diffusion effects and thus develop surface abundances similar to those that appear in the main-sequence, chemically peculiar group of A and B-type stars. We present the first high-resolution study of isotopic anomalies and chemical abundances in six Horizontal Branch B-type stars in globular clusters NGC 6397 and NGC 6752 and compare them to those observed in $\mathrm{HgMn}$ stars.

Methods. We obtained high-resolution (up to $R$ 115000 ) UVES spectra of a representative sample of six B-type stars (T183, T191, T193, B652, B2151, B2206) with sharp spectral lines $\left(v \sin i \leq 10 \mathrm{~km} \mathrm{~s}^{-1}\right)$. The stars T183 and B2151 were observed on two consecutive nights to examine potential spectrum variability. A detailed spectrum analysis was done relying on Kurucz ATLAS9 and ATLAS12 models. The spectra were analysed using the SYNTHE code to generate synthetic spectra.

Results. It is the first time an abundance analysis is performed for all elements for which spectral lines were detected in UVES spectra of Horizontal Branch B-type stars. Our study of these stars revealed no signs of He isotopic anomalies, which would produce a ${ }^{3} \mathrm{He} /{ }^{4} \mathrm{He}$ ratio different from the solar one. The isotopic anomaly of $\mathrm{Ca}$ is detected in all six studied stars. The chemical abundance analysis reveals an overabundance over the solar values of $\mathrm{P}, \mathrm{Ti}, \mathrm{Mn}, \mathrm{Fe}$, and $\mathrm{Y}$ and an overabundance over the cluster metallicity of $\mathrm{Mg}, \mathrm{Ca}$, and $\mathrm{Cr}$. This behaviour is very similar in all six stars of both clusters with a few exceptions: the Na abundance is larger by more than 1.4 dex than the cluster metallicity in B652, and by more than 0.8 dex greater than the cluster metallicity in B2206; the Co abundance is 1.0 dex over the solar abundance for $\mathrm{T} 191$, while $\mathrm{Zr}$ is overabundant over the solar abundance by 0.4 dex in B2206. No lines of $\mathrm{Hg}$ or other heavy elements were observed in the spectra. Weak emission lines of Ti II, similar to those frequently observed in HgMn stars have been discovered in one Horizontal Branch B-type star T191. Furthermore, we detected a radial velocity change of $0.9 \mathrm{~km} \mathrm{~s}^{-1}$ from one night to the next for T183 and $0.4 \mathrm{~km} \mathrm{~s}^{-1}$ for B2151. Further high-resolution observations are needed to examine whether both stars are spectroscopic binaries.
\end{abstract}

Key words. Galaxy: globular clusters: individual: NGC 6397, NGC 6752 - stars: Population II - stars: atmospheres stars: fundamental parameters - stars: abundances - stars: binaries: spectroscopic

\section{Introduction}

It is generally thought that isotopic anomalies are restricted to the chemically peculiar (CP) group of A and B-type stars. The study of isotopic anomalies of different chemical elements in chemically peculiar stars dates back to the 1960s when Sargent \& Jugaku (1961) detected an anomalously high concentration of ${ }^{3} \mathrm{He}$ in the chemically peculiar Bp star $3 \mathrm{Cen} \mathrm{A}$. Chemically peculiar A and B stars are main-sequence stars in the spectra of which lines of a number of elements appear abnormally strong or weak with respect to the bulk of normal A and B dwarfs of the same temperature. Altogether, the $\mathrm{CP}$ stars include the He-rich, He-weak, HgMn, Si, SrCrEu, and Am stars. The remarkable

* Based on observations obtained at the European Southern Observatory, Paranal, Chile (ESO programmes 076.D-0169(A) and 081.D-0498(A)). variety of elemental overabundances and depletions in the atmospheres of CP stars has been a puzzle for stellar spectroscopists for more than a century. Those Ap and Bp stars with significant dipolar or quadrupolar stellar magnetic fields have overabundances of $\mathrm{Si}, \mathrm{Ti}, \mathrm{Cr}, \mathrm{Sr}$, and rare earths, of up to several dex with underabundances of other elements such as He and $\mathrm{O}$. The Am stars show mild overabundances of most iron peak elements, but always abnormally weak Ca II lines. The most puzzling CP stars with amazing chemical and isotopic anomalies, so-called HgMn stars, belong to a group of most slowly rotating late B-type stars and show substantial overabundances of a number of elements, among them heavy ones, such as $\mathrm{Pt}, \mathrm{Au}, \mathrm{Hg}, \mathrm{Tl}$, and $\mathrm{Bi}$, and isotopic anomalies with patterns changing from one star to the next (Hubrig et al. 1999). In the magnetic Ap and Bp stars, the rare earth elements are among the most enhanced, up to 3-4 dex, whereas the heavy elements are overabundant in the 
atmospheres of $\mathrm{HgMn}$ stars by up to a million times compared to the solar system level.

The mechanisms responsible for producing the chemical peculiarities in upper main-sequence stars are still unclear. It is generally believed that the peculiar atmospheric abundances are a consequence of trace element diffusion from the stellar envelope. The anomalous abundances probably arise from competition between gravitational settling, radiative levitation, turbulent and convective mixing, and mixed and fractionated mass loss (e.g., Gonzales et al. 1995). However, the observed abundance distributions in these stars have never been completely explained by any calculation. The radiative diffusion mechanism alone cannot account for all abundance and isotopic anomalies (cf. Proffitt et al. 1999). Optical studies based on high-resolution spectra of $\mathrm{HgMn}$ stars concentrated on the behaviour of the $\mathrm{Hg}$ II $\lambda$ 3984, $\mathrm{Hg}$ I $\lambda$ 4385, and Hg II $\lambda 6149$ lines (e.g. Dolk et al. 2003). The high-resolution spectroscopic study of the isotopic composition of Pt (Hubrig et al. 1999) showed that this element behaves very much like $\mathrm{Hg}$, i.e. cooler stars exhibit a large relative overabundance of the heavy isotopes.

A new isotopic abundance anomaly in $\mathrm{HgMn}$ and Ap stars was discovered a few years ago by Castelli \& Hubrig (2004a) and Cowley \& Hubrig (2005). Observational evidence of large isotopic shifts in the infrared triplet of $\mathrm{Ca}$ II has been presented in a sample of HgMn stars (Castelli \& Hubrig 2004a). Calcium has six stable isotopes with mass numbers 40, 42, 43, 44, 46, and 48. The terrestrial isotopic mixture (in percent) is 96.941, $0.647,0.135,2.086,0.004$, and 0.187 , respectively (Anders \& Grevesse 1989). The maximum isotopic shifts between the isotopes ${ }^{40} \mathrm{Ca}$ and ${ }^{48} \mathrm{Ca}$ for the $\mathrm{Ca}$ II lines at $3968.47 \AA$ (H-line) and at $3933.64 \AA$ (K-line) is of the order of only $\sim 9 \mathrm{~m} \AA$ (MartenssonPendril et al. 1992), so that these lines are practically unaffected by possible calcium isotopic composition anomalies. On the other hand, the largest isotopic shift in the Ca II infrared triplet at 8498.023 $\AA$, 8542.091 transitions, is about $0.2 \AA$, according to Nörtershäuser et al. (1998).

As the origin of these isotopic anomalies is not known with certainty, it is important to investigate the isotopic behaviour in a variety of stars. The best candidates to search for similar chemical anomalies are slowly rotating Blue Horizontal Branch B-type (BHB) stars, which have similar $T_{\text {eff }}$ and $\log g$ to $\mathrm{HgMn}$ stars and shallow surface convective zones. The so-called hot BHB stars with $T_{\text {eff }}>11000 \mathrm{~K}$ display surface abundances that are significantly altered by diffusion effects in their atmospheres (e.g. Behr 2003). Stars in the horizontal branch (HB) have already passed the red giant branch (RGB) phase and are presently burning helium in their cores (Hoyle \& Schwarzschild 1955). In a number of globular clusters the HB shows "jumps" where stars appear brighter than theoretical expectations (e.g., Grundahl et al. 1999; Momany et al. 2002, 2004) or gaps, i.e. underpopulated regions along the HB (e.g. Sosin et al. 1997). While metal abundances roughly agree with the canonical cluster metallicity in cooler HB stars with $T_{\text {eff }}<11200 \mathrm{~K}$, most stars with $T_{\text {eff }} \gtrsim 11200 \mathrm{~K}$ are helium-depleted and show an increase in their metal content (e.g. Behr et al. 2000, 2003). Since almost all of these hot BHB stars are slowly rotating, it is expected that the observed abundance anomalies develop due to diffusion effects.

Similarly, a few mid-B spectral type stars are known to lie close to or on the horizontal branch, such as Feige 86, PHL 25, PHL 1434, and HD 135485. Abundance anomalies in these field BHB stars have been detected in various studies (e.g., Trundle et al. 2001; Bonifacio et al. 1995), which are most probably caused by radiative levitation and gravitational settling. Since April 2006, we have at our disposal a high-resolution spectrum of the Pop II halo B-type star Feige 86, for which we have been able to determine the isotopic structure of the $\mathrm{Hg}$ II $\lambda 3984$ line and a Ca II triplet shift of $0.13 \AA$ for the first time. The He I lines were previously studied by Bonifacio et al. (1995), and the detection of ${ }^{3} \mathrm{He}$ at $6678 \AA$ was announced thirty years ago by Hartoog (1979). Similar to Feige 86 and to many HgMn stars, BHB stars show overabundant $\mathrm{P}, \mathrm{Mn}, \mathrm{Fe} \mathrm{Ti}$, and $\mathrm{Cr}$ and underabundant He (e.g., Fabbian et al. 2005). However, the isotopic composition of $\mathrm{He}, \mathrm{Ca}$, and heavy elements in this type of stars has never been studied before.

In this work we focus our attention on the high-resolution study of the isotopic composition and chemical abundances of various elements in BHB stars in the globular clusters NGC 6397 and NGC 6752, and compare them to those determined previously in HgMn stars and the Pop II halo B-type star Feige 86. All previous spectroscopic studies of BHB stars made use of much lower spectral-resolution observation with the highest spectral resolution achieved with the HIRES spectrograph at the Keck 1 telescope (Behr 2003). The clusters NGC 6397 and NGC 6752 are the nearest globular clusters for which the phenomenon of radiative levitation of iron and heavy elements has been detected with certainty and for which a significant number of targets exist.

\section{Sample selection and observations}

Understandably, a study of chemical abundances, especially of the isotopic composition in slowly rotating BHB stars requires the availability of high-resolution spectra observed at a reasonable signal-to-noise ratio $(\mathrm{S} / \mathrm{N})$. Moderately high-resolution spectra with a resolving power of 30000 to 40000 of a sample of BHB stars in NGC 6397 and NGC 6752 were previously obtained at ESO in service mode with the VLT UV-Visual Echelle Spectrograph UVES at UT2 in the framework of programmes 65.L-0233(A) and 69.D-0220(A). We retrieved these spectra from the ESO archive, to select the most suitable candidates with the most sharp-lined spectra and strong appearance of iron group elements. Based on this study, we selected three BHB targets in NGC 6397 (T183, T191, and T193) and three BHB targets in NGC 6752 (B665, B2151, and B2206).

We then checked the position of these targets in the colour-magnitude diagrams of NGC 6397 and NGC6752. In Fig. 1 we show the dereddened $V,(U-V)$ and $V,(B-V)$ colour-magnitude diagrams of NGC6397 and NGC 6752 (Momany et al. 2003) and highlight the location of the selected BHB stars. The photometric data of NGC 6397 and NGC 6752 were obtained from ground-based $U B V$ observations collected in three different runs with the Wide-Field Imager (WFI) at the $2.2 \mathrm{~m}$ ESO-MPI telescope and were previously published in Momany et al. $(2002,2003)$. In contrast to the classical $V,(B-V)$ diagrams (left panels) where the colour saturation results in an almost vertical HB sequence, the ultraviolet diagrams (right panels) show interesting photometric features as stars become hotter along the HB.

Grundahl et al. (1999) identified a discontinuity in the Stromgren $(u, u-y)$ diagram at around $11500 \mathrm{~K}$. This discontinuity is easily detected in the upper right panel of Fig. 1 $[(U-V) \simeq-0.4]$, and marks a sudden divergence with respect to Zero-Age-Horizontal-Branch (ZAHB) models; i.e., stars between 11 500-20000 K appear brighter. This discontinuity has been attributed to radiative levitation of heavy elements that eventually enhance the atmospheric abundances of these BHB 


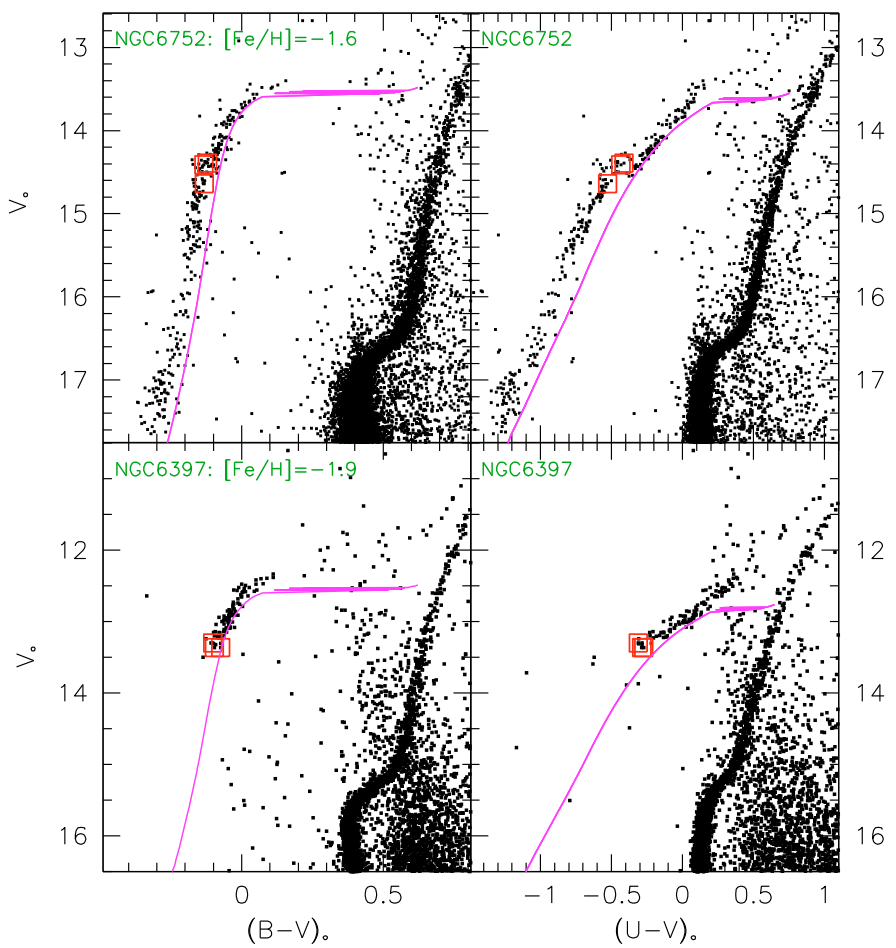

Fig. 1. Left panels display the classical $V,(B-V)$ colour-magnitude diagrams of NGC 6397 (lower left) and NGC 6752 (upper left). For both clusters, the studied BHB samples are plotted as open squares. Appropriate metallicity ZAHB models from Pietrinferni et al. (2006) are also plotted. Right panels display the respective ultraviolet $V,(U-$ $V)$ diagrams. In the case of NGC 6752, the location of the Grundahl et al. (1999) $u$-jump is easily distinguished at $(U-V) \simeq-0.4$.

stars. Noteworthy is that this $u$-discontinuity was proved to be present in all BHB clusters, always occurring at a similar temperature regardless of the cluster parameters (e.g., age, metallicity). In particular, Grundahl et al. find correspondence between the discontinuity and the so-called gravity jump (observed in both globular clusters and field stars). Furthermore, Recio-Blanco et al. (2002) show that the $u$-discontinuity might be related to the discontinuity in rotational velocity found around $T_{\mathrm{eff}} \geq 10000 \mathrm{~K}$ (see also Behr et al. 2000). Thus, the triggering of the Grundahl et al. $u$-jump traces the imprint of other anomalies (heavy element abundances, gravity, and rotational velocity). In this context, it is expected that the selected BHB stars are post- $u$ jump and therefore exhibit the above anomalies.

In the case of the NGC 6752 BHB star sample, this is a straightforward conclusion. Indeed, in the upper right panel of Fig. 1 we compare the BHB star distribution to that of an $[\mathrm{Fe} / \mathrm{H}]=-1.6 \mathrm{ZAHB}$ model (Pietrinferni et al. 2006) and show that all three selected stars are brighter than the ZAHB model. On the other hand, the horizontal branch of NGC 6397 is clearly less extended than that of NGC 6752, and even when the $V,(U-V)$ diagram is used, it is not easy to establish whether the selected stars are post- $u$ jump stars or not.

In Fig. 2 we overplot the $U,(U-V)$ diagram of the entire NGC 6752 HB sample (grey squares) and compare it with that of NGC 6397 (black squares). The NGC 6397 photometry has been shifted in such a way as to match the location of the NGC 6752 HB segment between the Grundahl et al. jump and the blue boundary of the instability strip. Keeping in mind that the $u$-jump has been found to be an ubiquitous feature always occurring at a similar temperature for all globular clusters

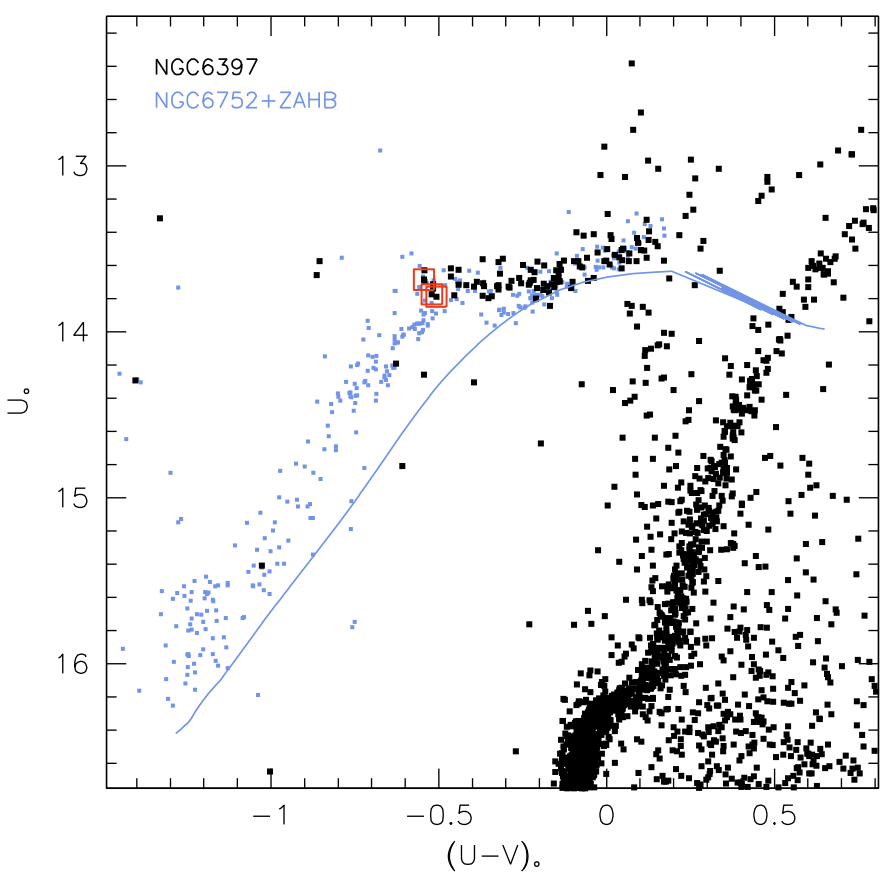

Fig. 2. A superposition of the NGC6397 $U,(U-V)$ colour-magnitude diagram on the HB location of NGC 6752. The ZAHB model has the metallicity of NGC 6752. The superposition shows that the three selected NGC 6397 BHB stars are most likely located on the post- $u$ jump HB segment.

(regardless of their metallicity and other properties), we conclude that such a superposition technique is helpful in highlighting the segmented nature of the globular cluster BHB stars (see e.g. Fig. 7 of Momany et al. 2004). Figure 2 shows that three selected NGC 6397 BHB stars are most likely located on the post- $u$ jump HB segment.

High-resolution spectra of three selected BHB stars in NGC 6397 (T183, T191, and T193) and three BHB stars in NGC 6752 (B665, B2151, and B2206) were observed on August 2 and 3, 2008 at ESO UVES at UT2. We used the UVES Dichroic 2 nonstandard setting $(437+700)$ to cover the ranges $\lambda \lambda 3758-4984,5074-6930$, and 7045-8904 $\AA$. For the star T183, the slit width was set to $0.3^{\prime \prime}$ for the red arm, corresponding to a resolving power of $\sim 115000$. The stars T191, T193, B652, B2151, and B2206 were observed with a slit width of $0.4^{\prime \prime}$ fot the red arm $(R \approx 95000)$. For the blue arm, for all stars, we used a slit width of $0.4^{\prime \prime}$ to achieve a resolving power of $\sim 80000$. The spectra were reduced by the UVES pipeline Data Reduction Software (version 4.3.0, the general description can be found in e.g. Ballester et al. 2000) and using standard IRAF routines. The $\mathrm{S} / \mathrm{N}$ of the obtained UVES spectra range is between $75-125$ in the near ultraviolet (UV) $(3800 \AA), 95-120$ at $4500 \AA$, and $60-110$ in the near-IR region containing the Ca II triplet lines. The stars T183 and B2151 were observed twice to examine potential spectrum variability, like the presence of chemical spots on the stellar surface or radial velocity variations due to the presence of a close companion. The numbers assigned to the stars in NGC 6397 and NGC 6752 follow Antony-Twarog \& Twarog (2000) and Buonanno et al. (1986), respectively. The observed stars are listed in Table 1. The first identification numbers (Star ID), positions, and $U B V$ photometry are from Momany et al. (2003), while the second identification numbers are from Buonanno et al. (1986) and Anthony-Twarog \& Twarog (2000) for NGC 6397 NGC 6752, respectively. Radial velocities 
Table 1. $U B V$ magnitudes of the NGC 6752 and NGC 6397 BHB star sample.

\begin{tabular}{rrrrrrrrrr}
\hline \hline Cluster & Star ID & Star ID & RA (J2000) & Dec (J2000) & $U$ & $B$ & $V$ & $\begin{array}{r}v_{\text {rad }} \\
{\left[\mathrm{km} \mathrm{s}^{-1}\right]}\end{array}$ & $\begin{array}{r}v \sin i \\
{\left[\mathrm{~km} \mathrm{~s}^{-1}\right]}\end{array}$ \\
\hline NGC 6397 & 30511 & T183-1 & $17: 40: 20.057$ & $-53: 41: 42.91$ & 13.944 & 13.866 & 13.858 & 21.1 & 8.5 \\
& & T183-2 & & & & & & 20.2 & \\
& 53234 & T191 & $17: 40: 56.022$ & $-53: 35: 07.39$ & 14.037 & 13.969 & 13.923 & 21.5 & 10.0 \\
& 52839 & T193 & $17: 40: 57.460$ & $-53: 35: 32.79$ & 14.014 & 13.954 & 13.922 & 24.0 & 8.0 \\
\hline NGC 6752 & 22300 & B652 & $19: 11: 31.490$ & $-59: 57: 52.48$ & 14.337 & 14.699 & 14.783 & -24.6 & 8.0 \\
& 28150 & B2151-1 & $19: 11: 01.965$ & $-59: 55: 30.90$ & 14.202 & 14.468 & 14.554 & -33.0 & 2.5 \\
& & B2151-2 & & & & & & -32.6 & \\
& 22949 & B2206 & $19: 11: 00.931$ & $-59: 57: 42.22$ & 14.202 & 14.459 & 14.532 & -26.3 & 2.5 \\
\hline
\end{tabular}

Table 2. Cluster parameters.

\begin{tabular}{clrll}
\hline \hline NGC & {$[\mathrm{Fe} / \mathrm{H}]$} & $(m-M)_{V}$ & $E(B-V)$ & $E(b-y)$ \\
\hline 6397 & $-1.91^{1,2}$ & $12.19^{1}$ & $0.179^{3}$ & $0.127^{3}$ \\
& & & \pm 0.003 & \pm 0.002 \\
6752 & $-1.54^{1}$ & $12.96^{1}$ & $0.04^{1}$ & \\
& $-1.61^{2}$ & & $0.05^{2}$ & \\
\hline
\end{tabular}

${ }^{1}$ Zinn (1985), ${ }^{2}$ Grundhal et al. (1999), ${ }^{3}$ Anthony-Twarog\& Twarog (2000).

$v_{\text {rad }}$ and values of $v \sin i$ are listed in the last two columns. The rotational velocity $v \sin i$ was derived from the comparison of observed and computed profiles, in particular using the line $\mathrm{Mg}$ II 4481.

\section{Abundance analysis}

A standard model atmosphere analysis was made using as starting models those computed with the ATLAS9 code (Kurucz 1993) assuming a solar chemical composition and, as final models, those computed with the ATLAS12 code (Kurucz 2005) for individual stellar abundances. We used Linux versions of the codes as described in Shordone et al. (2004) and Castelli (2005a).

\subsection{Atmospheric parameters}

The gravity was computed using its definition:

$g=g_{\odot} \frac{M}{R^{2}}$

where $g_{\odot}$ is the surface gravity of the sun and the mass, $M$, and radius, $R$, of the star are in solar units. According to the relation of radius to luminosity and therefore to bolometric magnitude, the gravity is given by

$$
\begin{aligned}
\log g= & \log g_{\odot}+\log \left(M / M_{\odot}\right)+0.4\left(M_{\mathrm{bol}}-M_{\mathrm{bol}, \odot}\right) \\
& +4 \log \left(T_{\mathrm{eff}} / T_{\odot}\right),
\end{aligned}
$$

where the bolometric magnitude is

$M_{\mathrm{bol}}=V-(m-M)_{V}+B C_{V}$

and $(m-M)_{V}$ the distance modulus, while $V$ is the visual magnitude corrected for reddening. We adopted $A(V)=3.1 E(B-V)$ and distance modulus and reddening listed in Table 2. Furthermore, we assumed $M_{\mathrm{bol}, \odot}=4.74$ (Bessell et al. 1998) and used the bolometric corrections $\mathrm{BC}_{V}$ computed by Bessell et al. (1998) for solar metallicity atmospheric models having zero microturbulent velocity. In fact, all the studied stars have $[\mathrm{Fe} / \mathrm{H}]$ nearly solar or even super-solar. As stellar total mass we assumed $M / M_{\odot}=0.6$. Behr (2003) estimates that an uncertainty of $0.06 M_{\odot}$ would produce an uncertainty of $0.04 \mathrm{dex}$ in $\log g$, caused by the uncertainties in temperature and metallicity. Under the assumption of solar parameters $T_{\text {eff }, \odot}=5777 \mathrm{~K}$, $\log g_{\odot}=4.4377$ we computed stellar gravities using the equation presented above. Because $T_{\text {eff }}$ is not known in advance, we adopted as $\log g$ the averages of the gravities obtained for $T_{\text {eff }}=11000 \mathrm{~K}, 11500 \mathrm{~K}, 12000 \mathrm{~K}$, and $12500 \mathrm{~K}$. They are presented in Table 3. For B652 we computed the gravities obtained from two different determinations of the visual magnitude, i.e. $V=14.70$ from Buonanno et al. (1986) and $V=14.743$ as quoted by Moehler et al. (2000). The corresponding maximum gravity difference amounts to $0.02 \mathrm{dex}$. An estimated error of $0.02 \mathrm{mag}$ in $E(B-V)$ affects the gravity by \pm 0.03 dex. However, the error of 0.003 dex as estimated by Antony-Twarog $\&$ Twarog (2000) induces a gravity error of only 0.004 dex. An error of $0.07 \mathrm{mag}$ in distance modulus corresponding to about $3.5 \%$ in the distance (Gratton et al. 2003) leads to an error of \pm 0.03 dex in the gravity.

Once the gravity was fixed for each star, we derived the temperature by forcing Fe I and Fe II abundances to be consistent. The resulting temperatures are given in Table 4 . Results are shown both from ATLAS9 models with $[\mathrm{M} / \mathrm{H}]=0.0$ and ATLAS12 models computed for individual abundances. The presented abundances $\log \epsilon(\mathrm{Fe} \mathrm{I})$ and $\log \epsilon(\mathrm{Fe}$ II $)$ are defined as $\log \left(N_{\text {elem }} / N_{\text {tot }}\right)$. The analysed Fe I and Fe II lines are listed in Table A.1 without any asterisk. We estimated that an uncertainty of $0.2 \mathrm{dex}$ in the gravity corresponds to a temperature error of $300 \mathrm{~K}$, which produces an uncertainty of 0.1 dex in the iron abundance. To estimate the temperature error related to a different choice of Fe I and Fe II, we measured the equivalent widths for a few other lines. They are marked with an asterisk in the Table A.1. The extended line number modifies the temperature by $100 \mathrm{~K}$.

For B-type stars, Balmer profiles can also be used to derive the atmospheric parameters, in particular the gravity, because Balmer lines are somewhat insensitive to the temperature for $T_{\text {eff }}>11000 \mathrm{~K}$. Using a $\chi^{2}$ method we fitted the observed $\mathrm{H} \alpha, \mathrm{H} \beta, \mathrm{H} \gamma$, and $\mathrm{H} \delta$ profiles, normalized to the continuum level and shifted to the laboratory wavelength, to the grid of Balmer profiles based on ATLAS9 model atmospheres computed for $[\mathrm{M} / \mathrm{H}]=0.0$ and microturbulent velocity $\xi=0.0 \mathrm{~km} \mathrm{~s}^{-1}$. Balmer lines were computed with the BALMER9 code (Kurucz 1993). Also in this case, we adopted solar metallicity rather than cluster metallicities ( -1.91 for NGC 6397 and -1.61 for NGC 6752) 
Table 3. The gravity determination.

\begin{tabular}{lccccccc}
\hline \hline & T183 & T191 & T193 & \multicolumn{2}{c}{ B652 } & B2151 & B2206 \\
& 13.881 & 13.954 & 13.963 & $\begin{array}{l}14.70 \\
\log g\end{array}$ & 14.743 & 14.45 & 14.30 \\
$T_{\text {eff }}$ & & & & & & & \\
\hline 11000 & 3.71 & 3.74 & 3.74 & 3.89 & 3.91 & 3.79 & 3.73 \\
11500 & 3.75 & 3.78 & 3.78 & 3.93 & 3.94 & 3.83 & 3.77 \\
12000 & 3.78 & 3.81 & 3.81 & 3.96 & 3.98 & 3.86 & 3.80 \\
12500 & 3.81 & 3.84 & 3.85 & 3.99 & 4.01 & 3.89 & 3.83 \\
average $\log g:$ & $3.76 \pm 0.04$ & $3.79 \pm 0.04$ & $3.79 \pm 0.04$ & $3.94 \pm 0.04$ & $3.96 \pm 0.04$ & $3.84 \pm 0.04$ & $3.78 \pm 0.04$ \\
\hline
\end{tabular}

Table 4. $T_{\text {eff }}$ derived from the Fe I and Fe II ionization equilibrium for a fixed gravity.

\begin{tabular}{|c|c|c|c|c|c|c|c|c|c|c|}
\hline Star & $\log g$ & $\bar{T} T_{\text {eff }}$ & $\overline{\log \epsilon(\mathrm{Fe} \mathrm{I})}$ & $\overline{l o g} \epsilon(\mathrm{Fe}$ II $)$ & $\overline{\overline{T_{\text {eff }}}}$ & $\overline{\log \epsilon(\mathrm{Fe} \mathrm{I})}$ & $\log \epsilon(\mathrm{Fe}$ II $)$ & $\overline{T_{\text {eff }}}$ & $\log g$ & Source \\
\hline & & \multicolumn{3}{|c|}{ ATLAS9 } & \multicolumn{3}{|c|}{ ATLAS12 } & \multicolumn{3}{|c|}{ Literature } \\
\hline T183 & 3.75 & 1114 & $T .1$ & -4.16 & 113 & $-4.19 \pm 0.0$ & -4.19 & $11950 \pm$ & & 1 \\
\hline T191 & 3.80 & 11770 & $-4.68 \pm 0.06$ & $-4.68 \pm 0.09$ & 1163 & $-4.70 \pm 0.06$ & $-4.69 \pm 0.10$ & & & \\
\hline T193 & 3.80 & 11480 & $-4.27 \pm 0.09$ & $-4.27 \pm 0.10$ & 1137 & $-4.29 \pm 0.09$ & $-4.29 \pm 0.10$ & $11430 \pm$ & & 1 \\
\hline B652 & 3.95 & 12250 & $-3.80 \pm 0.11$ & $-3.80 \pm 0.09$ & 1196 & $-3.86 \pm 0.11$ & $-3.85 \pm 0.09$ & $12500 \pm$ & $98 \pm 0.07$ & 2 \\
\hline B2151 & 3.85 & 11400 & $-4.18 \pm 0.05$ & $-4.18 \pm 0.11$ & 1131 & $-4.20 \pm 0.05$ & $-4.20 \pm 0.11$ & & & \\
\hline B2206 & 3.80 & 1129 & $-4.21 \pm 0.05$ & $-4.21 \pm 0.08$ & 1120 & $-4.22 \pm 0.05$ & $-4.22 \pm 0.08$ & & & \\
\hline
\end{tabular}

(1) de Boer et al. (1995), (2) Moehler et al. (2000) for $[\mathrm{M} / \mathrm{H}]=0.0$.

Table 5. Stellar parameters from Balmer profiles.

\begin{tabular}{ccccccccccc}
\hline \hline Star & \multicolumn{2}{c}{$\mathrm{H}_{\alpha}$} & \multicolumn{2}{c}{$\mathrm{H}_{\beta}$} & \multicolumn{2}{c}{$\mathrm{H}_{\gamma}$} & \multicolumn{3}{c}{$\mathrm{H}_{\delta}$} & \multicolumn{2}{c}{ Average } \\
& $T_{\text {eff }}$ & $\log g$ & $T_{\text {eff }}$ & $\log g$ & $T_{\text {eff }}$ & $\log g$ & $T_{\text {eff }}$ & $\log g$ & $T_{\text {eff }}$ & $\log g$ \\
\hline T 183 & 11300 & 3.9 & 10850 & 3.5 & 10750 & 3.6 & 11650 & 4.0 & $11137 \pm 361$ & $3.75 \pm 0.21$ \\
T 191 & 10350 & 3.4 & 12000 & 4.1 & 11750 & 3.9 & 11750 & 4.0 & $11462 \pm 650$ & $3.85 \pm 0.27$ \\
T 193 & 10400 & 3.4 & 11200 & 3.5 & 10950 & 3.6 & 10500 & 3.6 & $10763 \pm 327$ & $3.52 \pm 0.08$ \\
B 652 & 10750 & 3.6 & 11300 & 3.4 & 11000 & 3.6 & 10900 & 3.5 & $10988 \pm 201$ & $3.52 \pm 0.08$ \\
B2151 & 10450 & 3.6 & 11150 & 3.5 & 10500 & 3.6 & 10500 & 3.6 & $10650 \pm 289$ & $3.57 \pm 0.04$ \\
B2206 & 10450 & 3.6 & 10900 & 3.5 & 10650 & 3.6 & 10500 & 3.6 & $10625 \pm 175$ & $3.57 \pm 0.04$ \\
\hline
\end{tabular}

due to the nearly solar or even super-solar $[\mathrm{Fe} / \mathrm{H}]$ abundances of the studied stars. The parameters from Balmer profiles are listed in Table 5. The average temperatures and gravities are less than those listed in Table 4. The large standard deviations associated to the average temperatures partly stem from the low sensitivity of the Balmer profiles to temperature and partly from the difficulty in fixing the continuum level over the observed Balmer profiles caused by spectral imperfection in the UVES spectra related to the echelle orders. While the order combining does not affect the analysis of most of the lines much, it challenges the Balmer lines in that their extended wings span more than a single order. The discrepancy in the parameters derived with the two methods can also be ascribed to the solar metallicity used for computing Balmer profiles, while for almost all elements the abundances are different from the solar ones. In fact we tested that, for the same parameters, the Balmer lines from ATLAS12 models with individual abundances are weaker than those computed from ATLAS9 models with solar metallicity, so that ATLAS12 models would require a higher gravity and/or a lower $T_{\text {eff }}$ to fit the observed Balmer line profiles.

Since the first method is almost model independent as far as the gravity is concerned, we adopted as model parameters those presented in Table 4. The zero microturbulent velocity agrees with the value usually adopted for field B-type peculiar stars.
We did not find any indication that a higher value of $\xi$ would have been a better choice.

\subsection{Individual abundances in the stars}

Spectral lines belonging to elements He I, Mg II, P II, Ca II, Ti II, Cr II, Mn II, Fe I, Fe II, Ni II, and Y II have been detected in all stars, except for P II in T191 where instead lines of O I, Si II, and Co II were detected. The Si II lines were also observed in T183, while Na I was observed in B652 and B2206.

Equivalent widths were measured for Ti II, CrII, Fe I, and Fe II by integrating the residual intensities over the profiles. Also equivalent widths were measured for a few lines of P II and Y II, which were not too weak or not too affected by noise. For these lines the abundances were obtained with a Linux version (Castelli 2005b) of the WIDTH code (Kurucz 1993). For weak lines with no measurable equivalent widths, we derived the abundance from the line profiles with the synthetic spectrum method. Synthetic spectra were computed with a Linux version (Sbordone et al. 2004) of the SYNTHE code (Kurucz 2005). When no lines were observed for a given element, an upper abundance limit was fixed by reducing the intensity of the computed line at the level of the noise. The lines used to derive abundances are listed in Table A.1. Mean abundances for each 
Table 6. Abundances from ATLAS 9 models computed for $[\mathrm{M} / \mathrm{H}]=0.0$ and microturbulent velocity $\xi=0 \mathrm{~km} \mathrm{~s}{ }^{-1}$. At this step the helium abundance was assumed solar.

\begin{tabular}{|c|c|c|c|c|c|c|c|c|c|}
\hline Elem. & $\begin{array}{c}\mathrm{T} 183 \\
(11480,3.75)\end{array}$ & $\begin{array}{c}\mathrm{T} 191 \\
(11770,3.8)\end{array}$ & $\begin{array}{c}\text { T193 } \\
(11480,3.80)\end{array}$ & NGC 6397 & $\begin{array}{c}\text { B652 } \\
(12250,3.95)\end{array}$ & $\begin{array}{c}\text { B2151 } \\
(11400,3.85)\end{array}$ & $\begin{array}{c}\text { B2206 } \\
(11290,3.80)\end{array}$ & NGC 6752 & Sun \\
\hline C II & $\leq-5.02$ & $\leq-5.02$ & $\leq-5.02$ & -5.43 & $\leq-4.52$ & $\leq-4.52$ & $\leq-4.52$ & -5.13 & -3.52 \\
\hline $\mathrm{N}$ I & $\leq-5.12$ & $\leq-5.12$ & $\leq-5.12$ & -6.03 & $\leq-5.12$ & $\leq-5.82$ & $\leq-5.82$ & -5.73 & -4.12 \\
\hline O I & $\leq-5.21$ & -4.80 & $\leq-5.51$ & -5.12 & $\leq-5.51$ & $\leq-5.51$ & $\leq-5.51$ & -4.82 & -3.21 \\
\hline $\mathrm{Na} I$ & $\leq-6.71$ & $\leq-6.71$ & $\leq-6.71$ & -7.62 & -5.90 & $\leq-6.71$ & -6.45 & -7.32 & -5.71 \\
\hline Mg II & -6.11 & -6.38 & -6.00 & -6.37 & -5.68 & -5.75 & -5.71 & -6.07 & -4.46 \\
\hline Al I & $\leq-6.75$ & $\leq-6.75$ & $\leq-6.75$ & -7.48 & $\leq-7.15$ & $\leq-7.25$ & $\leq-7.00$ & -7.18 & -5.57 \\
\hline Si II & -7.49 & -6.76 & $\leq-7.80$ & -6.40 & $\leq-7.60$ & $\leq-7.70$ & $\leq-8.00$ & -6.10 & -4.49 \\
\hline P II & -5.40 & $\leq-6.59$ & $-5.43 \pm 0.10$ & -8.50 & $-4.89 \pm 0.25$ & -5.53 & $-5.60 \pm 0.10$ & -8.20 & -6.59 \\
\hline S II & $\leq-6.21$ & $\leq-6.41$ & $\leq-6.21$ & -6.62 & $\leq-6.21$ & $\leq-6.21$ & $\leq-6.21$ & -6.32 & -4.71 \\
\hline Ca II & -5.88 & -5.53 & -6.22 & -7.59 & -5.85 & -6.34 & -6.28 & -7.29 & -5.68 \\
\hline Sc II & $\leq-9.87$ & $\leq-9.87$ & $\leq-10.37$ & -10.78 & $\leq-9.87$ & $\leq-10.37$ & $\leq-10.37$ & -10.48 & -8.87 \\
\hline Ti II & $-6.34 \pm 0.09$ & $-5.31 \pm 0.11$ & $-6.69 \pm 0.09$ & -8.93 & $-7.00 \pm 0.11$ & $-6.87 \pm 0.07$ & $-6.88 \pm 0.07$ & -8.63 & -7.02 \\
\hline Cr II & $-7.05 \pm 0.05$ & $-6.43 \pm 0.03$ & $-6.54 \pm 0.05$ & -8.28 & $-6.81 \pm 0.04$ & $-6.88 \pm 0.03$ & $-6.80 \pm 0.05$ & -7.98 & -6.37 \\
\hline Mn II & -6.05 & -5.35 & -5.44 & -8.56 & -5.75 & -6.50 & -6.30 & -8.26 & -6.65 \\
\hline $\mathrm{Fe} I$ & $-4.17 \pm 0.09$ & $-4.68 \pm 0.06$ & $-4.27 \pm 0.09$ & -6.45 & $-3.80 \pm 0.11$ & $-4.18 \pm 0.05$ & $-4.21 \pm 0.05$ & -6.15 & -4.54 \\
\hline Fe II & $-4.17 \pm 0.11$ & $-4.68 \pm 0.09$ & $-4.27 \pm 0.10$ & -6.45 & $-3.80 \pm 0.09$ & $-4.18 \pm 0.11$ & $-4.21 \pm 0.08$ & -6.15 & -4.54 \\
\hline Co II & $\leq-7.92$ & -6.12 & $\leq-7.12$ & -9.03 & - & $\leq-7.92$ & $\leq-7.92$ & -8.73 & -7.12 \\
\hline $\mathrm{Ni}$ II & -6.14 & -6.00 & -6.25 & -7.70 & -6.30 & -6.60 & -6.60 & -7.40 & -5.79 \\
\hline Sr II & $\leq-10.57$ & $\leq-10.07$ & $\leq-10.07$ & -10.98 & $\leq-10.07$ & $\leq-10.57$ & $\leq-10.57$ & -10.68 & -9.07 \\
\hline Y II & -8.20 & -8.20 & $-8.15 \pm 0.05$ & -11.71 & $-7.43 \pm 0.12$ & -8.50 & $-8.34 \pm 0.08$ & -11.41 & -9.80 \\
\hline Zr II & -11.35 & -11.35 & -11.35 & -11.35 & -11.05 & -11.05 & -9.01 & -11.05 & -9.44 \\
\hline
\end{tabular}

Table 7. Final ATLAS12 abundances.

\begin{tabular}{lcccccccc}
\hline \hline Elem & T183 & T191 & T193 & NGC6397 & B652 & B2151 & B2206 & NGC 6752 \\
& $(11390,3.75)$ & $(11630,3.8)$ & $(11370,3.80)$ & & $(11960,3.95)$ & $(11310,3.85)$ & $(11200,3.80)$ & \\
\hline $\mathrm{He}$ & -2.07 & -1.76 & -2.10 & -1.11 & -2.15 & -2.30 & -2.30 & -1.11 \\
$\mathrm{O}$ & -5.21 & -4.85 & -5.51 & -5.12 & -5.50 & -5.50 & -5.50 & -4.82 \\
$\mathrm{Na}$ & -7.62 & -7.62 & -7.62 & -7.62 & -5.90 & -7.32 & -6.51 & -7.32 \\
$\mathrm{Mg}$ & -6.11 & -6.38 & -6.00 & -6.37 & -5.68 & -5.75 & -5.71 & -6.07 \\
$\mathrm{Al}$ & -7.48 & -7.48 & -7.48 & -7.48 & -7.18 & -7.25 & -7.18 & -7.18 \\
$\mathrm{Si}$ & -7.49 & -6.76 & -7.80 & -6.40 & -7.60 & -7.70 & -8.00 & -6.10 \\
$\mathrm{P}$ & -5.40 & -8.50 & $-5.42 \pm 0.10$ & -8.50 & $-4.87 \pm 0.25$ & -5.53 & $-5.57 \pm 0.11$ & -8.20 \\
$\mathrm{Ca}$ & -5.98 & -5.52 & -6.22 & -7.59 & -5.85 & -6.34 & -6.28 & -7.29 \\
$\mathrm{Ti}$ & $-6.37 \pm 0.08$ & $-5.38 \pm 0.10$ & $-6.73 \pm 0.09$ & -8.93 & $-7.07 \pm 0.11$ & $-6.91 \pm 0.07$ & $-6.91 \pm 0.07$ & -8.63 \\
$\mathrm{Cr}$ & $-7.06 \pm 0.05$ & $-6.45 \pm 0.03$ & $-6.56 \pm 0.05$ & -8.28 & $-6.86 \pm 0.04$ & $-6.90 \pm 0.03$ & $-6.82 \pm 0.05$ & -7.98 \\
$\mathrm{Mn}$ & -6.05 & -5.50 & -5.44 & -8.56 & -5.65 & -6.50 & -6.20 & -8.26 \\
$\mathrm{Fe}$ & $-4.19 \pm 0.09$ & $-4.69 \pm 0.10$ & $-4.29 \pm 0.10$ & -6.45 & $-3.85 \pm 0.11$ & $-4.20 \pm 0.11$ & $-4.22 \pm 0.08$ & -6.15 \\
$\mathrm{Co}$ & -9.03 & -6.22 & -9.03 & -9.03 & -8.73 & -8.73 & -8.73 & -8.73 \\
$\mathrm{Ni}$ & -6.14 & -6.00 & -6.25 & -7.70 & -6.30 & -6.60 & -6.60 & -7.40 \\
$\mathrm{Y}$ & -8.20 & -8.20 & $-8.19 \pm 0.05$ & -11.71 & $-7.52 \pm 0.12$ & -8.50 & $-8.38 \pm 0.8$ & -11.41 \\
$\mathrm{Zr}$ & -11.35 & -11.35 & -11.35 & -11.35 & -11.05 & -11.05 & -9.05 & -11.05 \\
\hline
\end{tabular}

species based on ATLAS9 model atmospheres are summarised in Table 6. In this table, errors are only given for lines with measured equivalent widths, and no errors are presented for lines with abundances from comparison of observed and computed profiles using synthetic spectrum method. We did not use equivalent widths for Mn II because Mn is affected by hyperfine structure. When available, the hyperfine components are considered in the synthetic spectrum.

ATLAS9 models were not used to derive the He abundance. As a matter of fact, inaccurate results are expected due to the inconsistency between the populations from the ATLAS9 model computed for a solar $\mathrm{He} / \mathrm{H}$ ratio and the populations obtained by SYNTHE for modified $\mathrm{He} / \mathrm{H}$ values. The helium abundance was derived later by comparing the observed He I profiles with profiles computed from the ATLAS12 models used for the individual stellar abundances.

We applied an iterative procedure. For each star, an ATLAS12 model was computed after adopting the ATLAS9 parameters listed in Table 4 and the abundances listed in Table 6 for elements having observed lines in the spectra. For elements without observed lines, the abundance was assumed to be equal to the metallicity of the cluster provided that the abundance limit given in Table 6 exceeds the cluster metallicity. As for oxygen and silicon, the abundance limit was otherwise adopted. New abundances from equivalent widths and line profiles were determined with ATLAS12. Keeping the model independent gravity fixed, a new $T_{\text {eff }}$ was determined from the condition that the Fe I and Fe II abundances concur. The iteration was continued until ATLAS12 abundances and abundances derived from equivalent widths and line profiles agreed within $0.01 \mathrm{dex}$.

In Table 4, in Cols. 6-8, we present the ATLAS12 final parameters and the corresponding iron abundances. In Table 7 we list the final abundances used for computing the ATLAS12 models. For elements not listed in the table the abundances are those of the cluster, i.e. [-1.91] for NGC 6397 and [-1.61] for NGC 6752. The elemental abundances are given as 
Table 8. Final abundances $\log \left(N_{\text {elem }} / N_{\text {tot }}\right)$ relative to the solar abundances from Grevesse \& Sauval (1998) as derived from ATLAS12 models.

\begin{tabular}{|c|c|c|c|c|c|c|}
\hline \multirow[b]{2}{*}{ Elem. } & \multicolumn{3}{|c|}{ NGC 6397} & \multicolumn{3}{|c|}{ "NGC 6752} \\
\hline & $\begin{array}{c}\mathrm{T} 183 \\
(11390,3.75)\end{array}$ & $\begin{array}{c}\mathrm{T} 191 \\
(11630,3.80)\end{array}$ & $\begin{array}{c}\mathrm{T} 193 \\
(11370,3.80)\end{array}$ & $\begin{array}{c}\mathrm{B} 652 \\
(11960,3.95)\end{array}$ & $\begin{array}{c}\text { B2151 } \\
(11310,3.85)\end{array}$ & $\begin{array}{c}\text { B2206 } \\
(11200,3.80)\end{array}$ \\
\hline$[\mathrm{M} / \mathrm{H}]$ & & {$[-1.91]$} & & & {$[-1.61]$} & \\
\hline $\mathrm{He}$ & {$[-0.96]$} & {$[-0.65]$} & {$[-0.99]$} & {$[-1.04]$} & {$[-1.19]$} & {$[-1.19]$} \\
\hline O I & $\leq[-2.00]$ & {$[-1.64]$} & $\leq[-2.30]$ & $\leq[-2.29]$ & $\leq[-2.29]$ & $\leq[-2.29]$ \\
\hline $\mathrm{NaI}$ & & & & {$[-0.19]$} & & $-[0.80]$ \\
\hline Mg II & {$[-1.65]$} & {$[-1.92]$} & {$[-1.54]$} & {$[-1.22]$} & {$[-1.29]$} & {$[-1.25]$} \\
\hline $\mathrm{Al}$ I & & & & & $\leq[-1.68]$ & \\
\hline Si II & {$[-3.00]$} & {$[-2.27]$} & $\leq[-3.31]$ & $\leq[-3.11]$ & $\leq[-3.21$ & $\leq[-3.51]]$ \\
\hline P II & {$[+1.19]$} & & {$[+1.17]$} & {$[+1.72]$} & {$[+1.06]$} & {$[+1.02]$} \\
\hline Ca II & {$[-0.30]$} & {$[+0.16]$} & {$[-0.54]$} & {$[-0.17]$} & {$[-0.66]$} & {$[-0.60]$} \\
\hline Ti II & {$[+0.65]$} & {$[+1.64]$} & {$[+0.29]$} & {$[-0.05]$} & {$[+0.11]$} & {$[+0.11]$} \\
\hline Cr II & {$[-0.69]$} & {$[-0.08]$} & {$[-0.19]$} & {$[-0.49]$} & {$[-0.53]$} & {$[-0.45]$} \\
\hline Mn II & {$[+0.60]$} & {$[+1.15]$} & {$[+1.21]$} & {$[+1.00]$} & {$[+0.15]$} & {$[+0.45]$} \\
\hline $\mathrm{Fe}$ I-Fe II & {$[+0.35]$} & {$[-0.15]$} & {$[+0.25]$} & {$[+0.69]$} & {$[+0.34]$} & {$[+0.32]$} \\
\hline Co II & & {$[+0.90]$} & & & & \\
\hline Ni II & {$[-0.35]$} & {$[-0.21]$} & {$[-0.56]$} & {$[-0.51]$} & {$[-0.81]$} & {$[-0.81]$} \\
\hline Y II & {$[+1.60]$} & [+1.60] & {$[+1.61]$} & {$[+2.28]$} & {$[+1.30]$} & {$[+1.42]$} \\
\hline Zr II & & & & & & {$[+0.39]$} \\
\hline
\end{tabular}

$\log \left(N_{\text {elem }} / N_{\text {tot }}\right)$. Similar to the entries in Table 6, errors are given only for lines with measured equivalent widths, and no errors are presented for lines with abundances from comparison of observed and computed profiles using the synthetic spectrum method. For Mn I we did not use equivalent widths because Mn is affected by hyperfine structure. The hyperfine components are considered in the synthetic spectrum. Finally, Table 8 summarises the peculiar abundances relative to the solar values.

The He underabundance was the only anomaly measured in cluster BHB stars (Greenstein et al. 1967) for quite a long time. This underabundance is generally attributed to gravitational settling. Only the He I lines at $\lambda \lambda$ 4026.2, 4471.5, 5875.7, and $6678.15 \AA$ are observable in all stars. The lines at $\lambda \lambda 3819.6$, 4120.8 , and $4387.93 \AA$ can be observed as broad weak absorptions, whereas He I lines at $\lambda \lambda$ 3867.5, 4143.76, 4713.2, and $4921.93 \AA$ are either not observed or are components of blends with Fe I and Fe II lines. The He I line data are those listed in Appendix A of Castelli \& Hubrig (2004b) and the computation of the line profiles is described in the same paper. In all stars, except for B652, all He I lines can be reproduced by the same abundance, so that there are no signs of helium vertical abundance stratification. In B652 the observed He I profile at $5875.7 \AA$ requires an abundance 0.2 dex larger than that derived from the other lines. We could explain that this discrepancy is caused either by NLTE effects or by vertical abundance stratification. But this last case would imply an increase in the abundance toward the upper atmospheric layers in contrast to what happens in field stars that show shallow line cores and extended wings.

Elements more abundant than the cluster metallicity in all BHB stars are $\mathrm{Ca}, \mathrm{Ti}, \mathrm{Cr}, \mathrm{Mn}, \mathrm{Fe}, \mathrm{Ni}$, and $\mathrm{Y}$. Phosphorus is overabundant in all stars except for T191 where it was not observed. $\mathrm{Na}$ is overabundant in both B652 and B2206, Co is overabundant only in T191 and Zr is overabundant in B2206.

On the other hand and in addition to He, which is always underabundant, Si also always appears less abundant than the cluster metallicity (see e.g. Fig. 4). Oxygen is also underabundant, except for T191, which shows oxygen overabundance by about 0.3 dex. The Si abundance can be best determined in T183 and T191 thanks to the presence of weak spectral lines. Furthermore, we note that $\mathrm{Al}$ is less abundant than cluster metallicity in
B2151. No definite differences were observed between the abundances of neutral and ionized states of studied elements, indicating the absence of vertical elemental stratifications in the atmospheres of BHB stars. We note, however, that, apart from Fe I lines, all other lines of neutral states like those of Cr I and Mn I are extremely weak and cannot be used for the abundance analysis.

In Fig. 3 we present final ATLAS12 abundances $\log \left(N_{\text {elem }} / N_{\text {tot }}\right)$ relative to the solar abundances for each star. The top panels present abundances of the typical $\mathrm{HgMn}$ star HD 175640 of a similar temperature with $T_{\text {eff }}=12000 \mathrm{~K}$ (Castelli \& Hubrig 2004b). The largest abundance dispersions within the cluster are found for elements $\mathrm{Si}, \mathrm{P}, \mathrm{Ti}, \mathrm{Mn}$, and $\mathrm{Co}$ in NGC 6397. In the cluster NGC 6752 the largest abundance dispersions are found for $\mathrm{Na}, \mathrm{P}, \mathrm{Mn}, \mathrm{Y}$, and $\mathrm{Zr}$. Comparing the abundances between all studied stars, we note that the star T191 clearly stands out in the sample due to the underabundance of phosphorus and strong overabundance of titanium and cobalt. As we show in Sect. 4.2, that it is also the only star which exhibits emissions in Ti II lines in the region 6000-6110 $\mathrm{A}$. This star should certainly be studied in more detail in future follow-up studies. No evident trends in elemental abundances with fundamental parameters $T_{\text {eff }}, \log g$, and $v \sin i$ were found in our sample stars, although it is possible that the yttrium abundance is slightly increasing with $T_{\text {eff. }}$. Also the largest titanium overabundance was found in T191, which rotates slightly faster than other sample stars. On the other hand, the existence of trends should be examined further by observations of a larger sample of BHB stars, as our sample stars have a rather narrow range of parameters. The comparison of abundance patterns between BHB stars and the typical HgMn star HD 175640 shows somewhat similar trends, apart from the low metallicity of the major part of the studied elements. To our knowledge, no other abundance study of any hot BHB star in NGC 6397 has been performed in the past. The overabundance of iron and normal magnesium abundance are reported by Moehler et al. (1999), who studied 19 BHB stars in NGC 6752 using low-resolution spectra obtained with the ESO $1.52 \mathrm{~m}$ telescope. While the authors find an enrichment of iron by a factor of 50 on average with respect to cluster abundance and a magnesium 

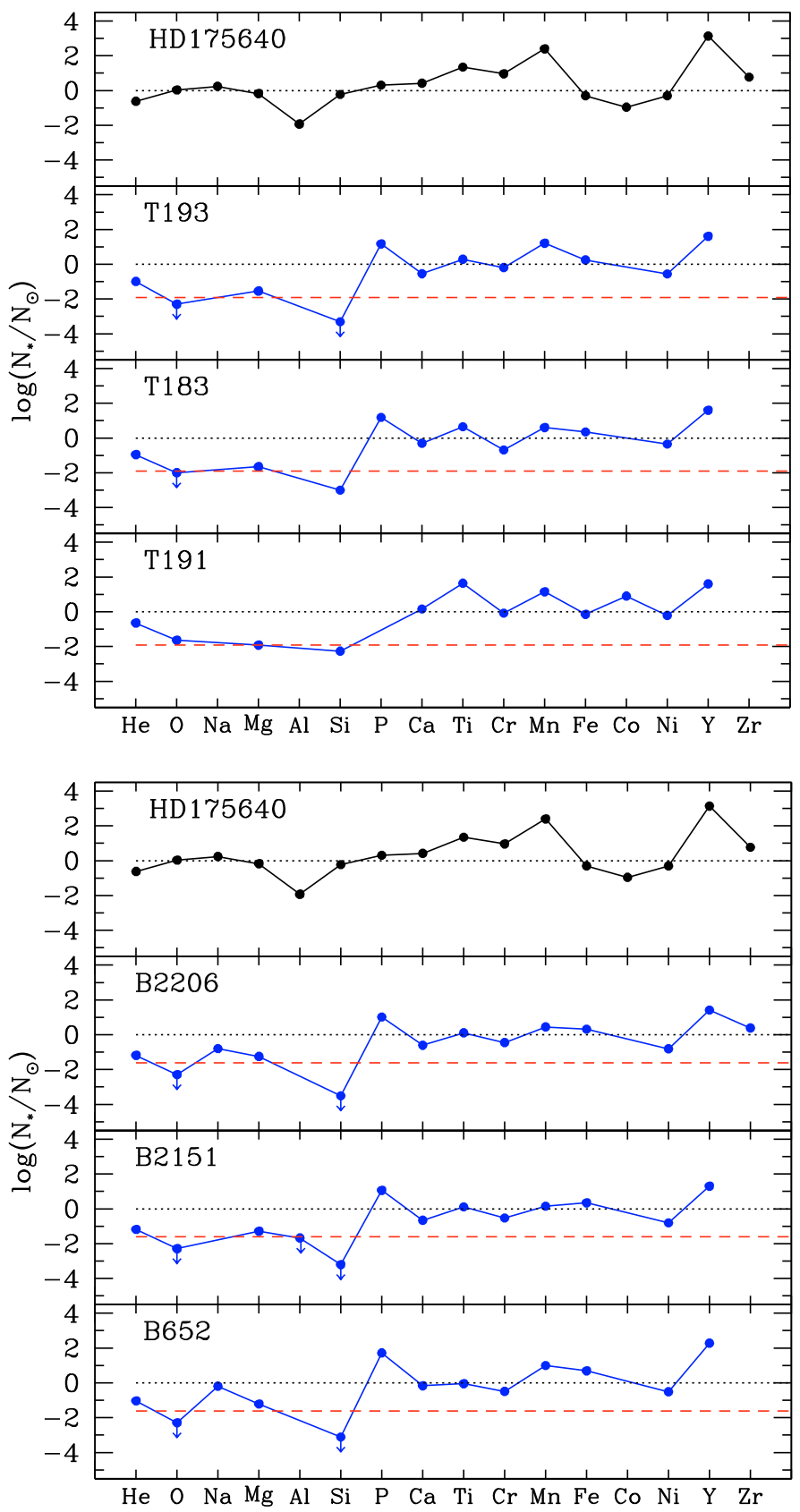

Fig. 3. Final ATLAS12 abundances $\log \left(N_{\text {elem }} / N_{\text {tot }}\right)$ for the members NGC 6397 and of NGC 6752 relative to the solar abundances (presented by the dotted horizontal line). The error bars cannot be presented on these plots as they are smaller than the plotted symbols. The dashed line indicates the cluster abundances. BHB stars are shown in the order of increasing $T_{\text {eff }}$ from top to bottom. The elements for which only an upper limit of the abundance is available are indicated by a downward arrow. The upper panels present abundances of the typical $\mathrm{HgMn}$ star HD 175640.

abundance consistent with the cluster metallicity in BHB stars with $T_{\text {eff }}>11000 \mathrm{~K}$, our abundance results show an enrichment of iron by an average factor of 100 and enrichment of magnesium by a factor of $\sim 2$, for both NGC 6397 and NGC 6752. Only for T191 in NGC 6397 is the magnesium abundance consistent with the cluster abundance. These differences in the abundance analyses are possibly explained by the higher resolution of our spectra and advanced methods of abundance determinations using the now available ATLAS12 models. Glaspey et al. (1989)

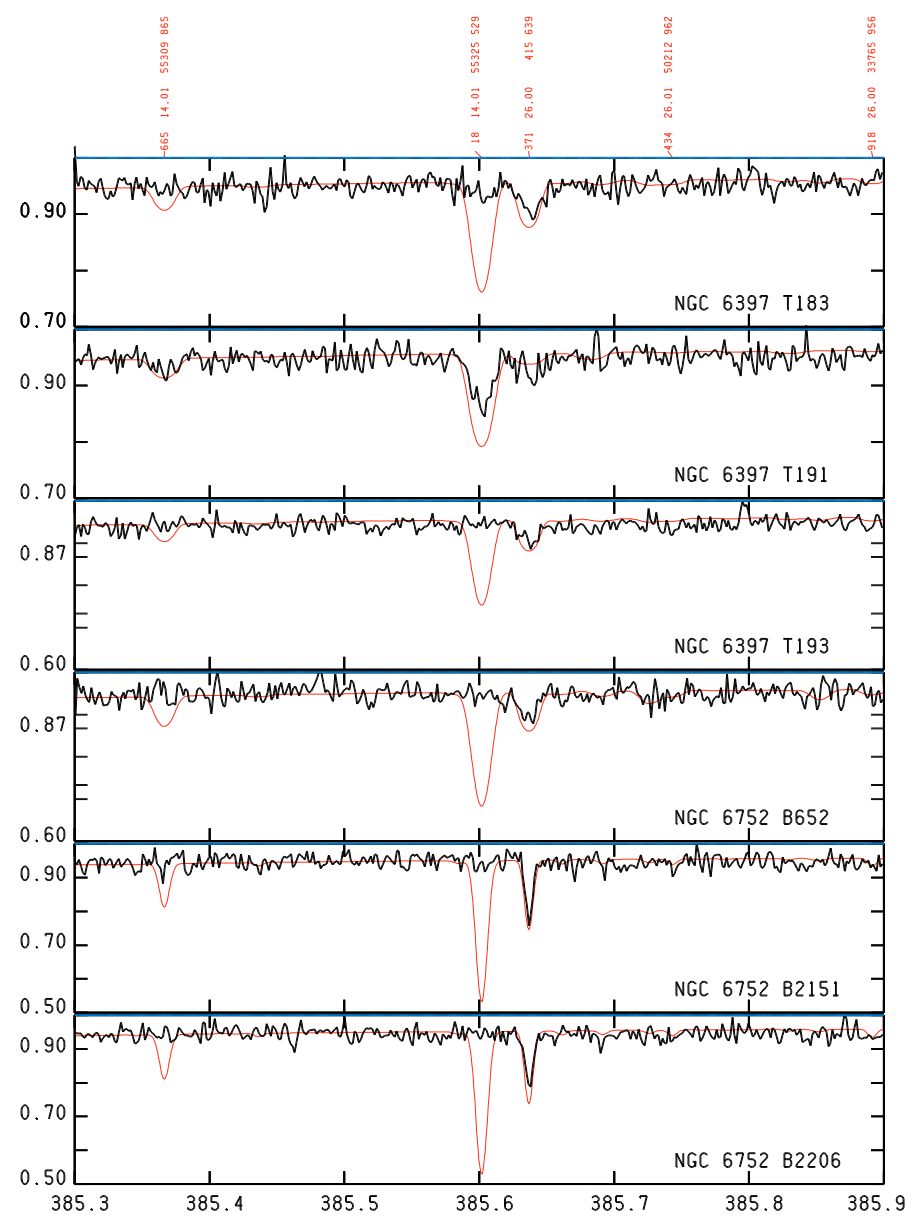

Fig. 4. Si II 3856.18 computed with the abundance of the clusters (thin line), i.e. -6.40 for NGC 6397 and -6.10 for NGC 6752 .

studied in addition to iron and magnesium silicon and phosphorus in a somewhat hotter BHB star, CL1083, in NGC 6397 with parameters $T_{\text {eff }}=16000 \mathrm{~K}$ and $\log g=4.0$. While silicon abundance was found consistent with the cluster abundance, no phosphorus lines could be identified in the spectrum of this star.

Interestingly, the stars T183 and B2151, which were observed on two consecutive nights to examine potential spectrum variability show small changes in radial velocities from one night to the next. The values $v_{\text {rad }}$ in Table 1 are heliocentric radial velocities measured by cross-correlations. The zero point has been established by using our previous observations of $\mathrm{HgMn}$ stars with FEROS. Dispersion of the subregions indicated an error less than $0.2 \mathrm{~km} \mathrm{~s}^{-1}$, and the total absolute error including calibrations and radial velocities of the template was less than $0.4 \mathrm{~km} \mathrm{~s}^{-1}$. Likewise, the radial velocities were improved by using telluric lines, so that relative velocites are more precise than $0.2-0.3 \mathrm{~km} \mathrm{~s}^{-1}$. The star T183 shows a radial velocity change of $0.9 \mathrm{~km} \mathrm{~s}^{-1}$ from one night to the next, whereas the shift in radial velocity for B2151 amounts to the value $0.4 \mathrm{~km} \mathrm{~s}^{-1}$, which is comparable to the measurement accuracy. We conclude that it is possible that T183 is a spectroscopic binary, although further spectroscopic observations are highly desirable.

In all stars, complex interstellar structures are found close to the position of $\mathrm{Ca}$ II at $3933.664 \AA$ and $3968.469 \AA$ and of Na I at 5889.950 $\AA$ and 5895.924 $\AA$. The interstellar structure affects the blue wings of the Ca II lines in T181, T191, and T193. The interstellar lines are blueshifted with respect to the stellar 


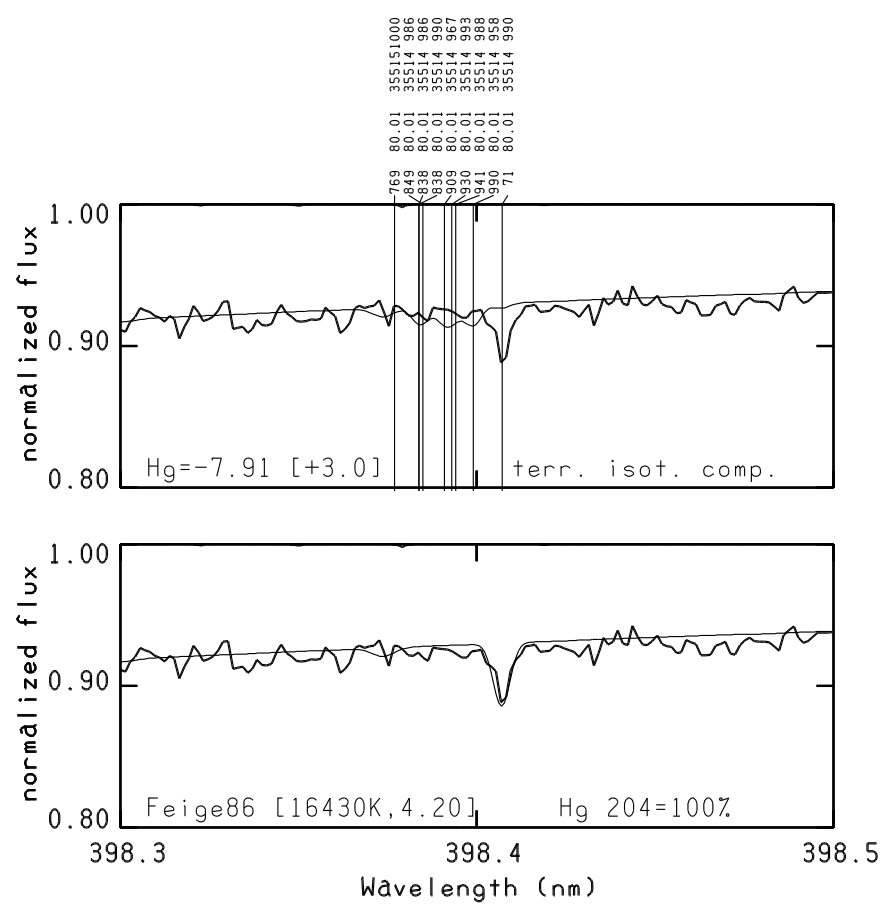

Fig. 5. The observation of ${ }^{204} \mathrm{Hg}$ in the spectrum of Feige 86. The upper panel shows the observed UVES spectrum and the synthetic spectrum (thin line) when assuming terrestrial isotopic composition for all isotopes. In the bottom panel, we show the synthetic spectrum calculated by assuming $\mathrm{Hg}$ II is present in the atmosphere entirely in the form of the heaviest stable isotope ${ }^{204} \mathrm{Hg}$.

wavelengths in T181, T191, and T193, while they are red-shifted in B652, B2151, and B2206.

\section{Presence of isotopes and emission lines}

\subsection{Isotopes}

The chemical peculiarities in HgMn stars have already been studied in numerous papers in the past. The studies reveal that $\mathrm{HgMn}$ stars of similar age, as inferred from their atmospheric parameters, can have widely different isotopic anomalies and chemical abundances indicating that the $\mathrm{HgMn}$ star phenomenon is a result of a complex interplay between a number of physical mechanisms (e.g. Dolk et al. 2003 ). The Pop II halo B-type star Feige 86, similar to HgMn stars and BHB stars, shows overabundant $\mathrm{P}, \mathrm{Mn}, \mathrm{Fe} \mathrm{Ti}, \mathrm{Cr}$, and underabundant $\mathrm{He}$. Apart from the $\mathrm{He}$ isotopic anomaly discussed by Bonifacio et al. (1995) significant $\mathrm{Ca}, \mathrm{Pt}$, and $\mathrm{Hg}$ isotopic anomalies have recently been discovered in a UVES spectrum of this star (Castelli \& Hubrig, in preparation). In Fig. 5 we present the observed $\mathrm{Hg}$ II $\lambda 3984$ line with the synthetic spectrum computed assuming $\mathrm{Hg}$ II being present in the atmosphere entirely in form of the heaviest stable isotope ${ }^{204} \mathrm{Hg}$.

Our study of BHB stars revealed no signs of He isotopic anomalies that would produce ${ }^{3} \mathrm{He} /{ }^{4} \mathrm{He}$ ratio different from the solar one. The largest isotopic shift of $0.50 \AA$ separates ${ }^{4} \mathrm{He}$ at $6678.152 \AA$ from ${ }^{3} \mathrm{He}$ at $6678.652 \AA$. The ${ }^{3} \mathrm{He}$ isotope was not observed in any star. In B2151 the line at $6678.152 \AA$ is so weak that an eventual isotope would be merged in the noise. In one BHB star, T191, the line He I $4471.5 \AA$ appears blueshifted by
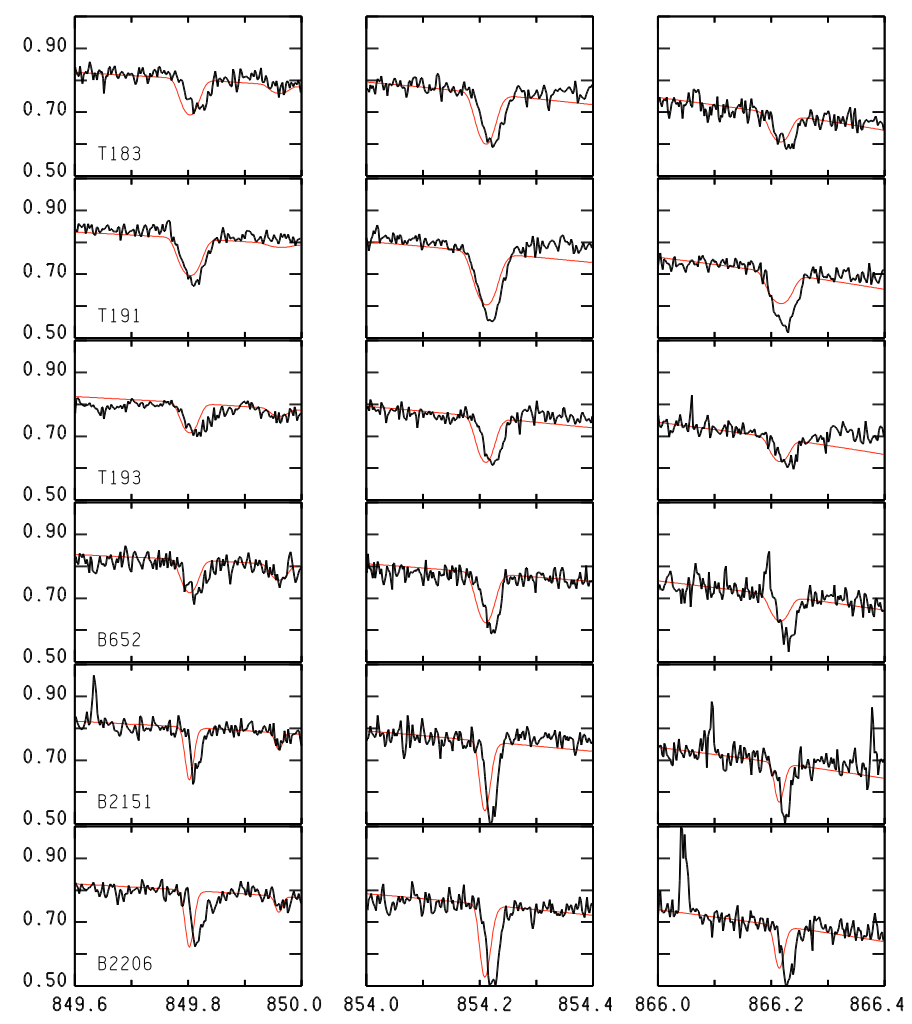

Fig. 6. Observed Ca II triplet shifts in all studied stars. The computed profiles assuming the terrestrial isotopic mixture are presented by thin lines.

$-0.04 \AA$, while the line He I $4713.2 \AA$ was found redshifted by $+0.06 \AA$.

In many $\mathrm{CP}$ stars isotopic structures of $\mathrm{Ga}$ and $\mathrm{Ba}$ lines, as well as isotopic anomalous ratios for $\mathrm{He} \mathrm{I,} \mathrm{Ca} \mathrm{II,} \mathrm{Pt} \mathrm{II,} \mathrm{Hg}$ I, and $\mathrm{Hg}$ II lines, were observed in the past. Our search for the presence of isotopic structures in the studied stars has led to recognise the presence of an isotopic anomaly only for the Ca II infrared triplet. In all these BHB stars, the Ca II triplet lines are redshifted by a different amount with the lowest shift of 0.06-0.07 $\AA$ in T191 to $0.13 \AA$ in T183 and T193. We measure in B2206 a shift of $0.14 \AA$ in the Ca II infrared triplet line 8662.141, but the Ca profiles in this star are rather asymmetrical leading to a lower accuracy of these measurements. The accuracy of the wavelength calibration in the region of the Ca triplet is $0.0018 \AA$ (rms of the thorium lines). On the other hand, the uncertainty of the effective wavelength of the Ca triplet lines also depends on the $\mathrm{S} / \mathrm{N}$ ratio of the spectra and on the shape of the line profile. Only for symmetrical, unblended lines can the wavelengths be measured to an accuracy better than $0.01 \AA$. For asymmetrical lines, differences up to $0.03 \AA$ may occur even for repeated measurements of the same line. In Fig. 6 we present the comparison of the observed Ca II infrared triplet lines in the studied BHB stars with the Ca II triplet lines computed assuming the terrestrial isotopic mixture. In the stars with the lowest $v \sin i$ values, B2151 and B2206, some kind of structure is noticeable in the $\mathrm{Ca}$ II infrared triplet lines. However, because of the rather low $\mathrm{S} / \mathrm{N}$ of our spectra, it is premature to suggest that this structure presents the $\mathrm{Ca}$ II isotopic structure, and the observed features in the line profiles could actually be produced by noise. Future studies of these Ca II infrared triplet lines in B2151 and B2206 
Table 9. $\Delta \lambda$ shift in the positions of the Ca II lines of the infrared triplet.

\begin{tabular}{|c|c|c|c|c|c|c|}
\hline$\lambda(\AA)$ & \multicolumn{6}{|c|}{$\Delta \lambda(\AA)$} \\
\hline 8498.023 & 0.13 & 0.07 & 0.13 & 0.10 & 0.11 & 0.11 \\
\hline 8542.091 & 0.11 & 0.06 & 0.13 & 0.11 & 0.11 & 0.11 \\
\hline 8662.141 & 0.11 & 0.07 & 0.13 & 0.12 & 0.11 & 0.14 \\
\hline
\end{tabular}

would require much higher $\mathrm{S} / \mathrm{N}$ spectroscopic material. Table 9 lists the amount of the observed shifts.

In Fig. 7 we present $\mathrm{Ca}$ isotopic shifts for $\mathrm{Ca}$ II triplet lines $(\lambda 8498, \lambda 8542$ and $\lambda 8662)$ for each BHB star versus $T_{\text {eff }}$ and $\log g$. We see no indication that $\mathrm{Ca}$ isotopic anomalies are related to the atmospheric parameters of our stars. Recently, Cowley et al. (2007) have studied a potential temperature correlation of the $\mathrm{Ca}$ isotopic anomaly in magnetic Ap stars and $\mathrm{HgMn}$ stars. They notice that there may be a weak correlation with temperature for $\mathrm{HgMn}$ stars in the sense that hotter stars show larger isotopic shifts. Clearly, more measurements of Ca II triplet lines in a larger stellar sample are necessary to establish whether a similar trend exists for BHB stars.

No comparison of isotopic structure with that of $\mathrm{HgMn}$ stars can be done for other elements, because lines of $\mathrm{Ga}, \mathrm{Ba}, \mathrm{Au}, \mathrm{Pt}$, and $\mathrm{Hg}$ were not observed in our BHB sample stars.

\subsection{Emissions}

The phenomenon of weak emission lines at optical wavelengths for main-sequence B-type stars and $\mathrm{HgMn}$ stars has been noted from observations of relatively few stars. Presently, explanations of this phenomenon have been put forward in the context of the non-LTE line formation (Sigut 2001) and possible fluorescence mechanisms (Wahlgren \& Hubrig 2000). No obvious correlations have been noted for emission in terms of stellar parameters of studied B-type stars. In CP stars emissions have mainly been detected in Mn II, mult.13, Cr II, $\lambda \lambda$ 6182.340, 6285.601, 6299.534, 6309.669, 6324.198, 6551.373, 6585.241, 6592.341, 6636.427; Ti II, $\lambda \lambda$ 6001.395, 6029.271, 6040.120, 6042.201, 6106.471, Fe II, $\lambda \lambda$ 6082.882, 6165.893, 6214.948, 6291.830, 6375.792, 8490.079, 8609.506, 8636.587, 8740.349, 8833.963 (e.g., Wahlgren \& Hubrig 2000; Wahlgren \& Hubrig 2004; Castelli \& Hubrig 2004b).

In T191, a few emissions are detected in Ti II lines in the region $6000-6110 \AA$ (see e.g. Fig. 8). It is quite possible that the line Mn II $\lambda 6125.863$ in B652 shows a weak emission, but the low $\mathrm{S} / \mathrm{N}$ ratio does not allow us to consider this line as a detection. It is also possible that very weak emissions are visible in the spectrum of T183 in lines Mn II $\lambda \lambda$ 6122.434, 6125.863, $6128.734,6130.796$ (see Fig. 9). For these two stars additional, much higher $\mathrm{S} / \mathrm{N}$ observations are required to confirm the presence of weak emissions in Mn II lines.

No Mn II emission lines were detected in T191 and T193, although the observed lines belonging to mult.13 are much weaker than computed. No emission lines were found in other BHB stars.

\section{Discussion}

The main result of the presented observations of two globular clusters is the overabundance over the solar values of $\mathrm{P}, \mathrm{Ti}, \mathrm{Mn}$, $\mathrm{Fe}$, and $\mathrm{Y}$, and the overabundance over the cluster metallicity of $\mathrm{Mg}, \mathrm{Ca}$, and $\mathrm{Cr}$. This behaviour is the same in all six studied stars of both clusters, apart from phosphorus, which was not observed in T191. Furthermore, the Na abundance is larger by more than 1.4 dex than the cluster metallicity in B652, and by more than 0.8 dex larger than the cluster metallicity in B2206. The Co abundance is 1.0 dex over the solar abundance for T191, while $\mathrm{Zr}$ is overabundant over the solar abundance by 0.4 dex in B2206. The Pop II halo B-type star Feige 86, similar to BHB stars shows overabundant $\mathrm{P}, \mathrm{Ti}, \mathrm{Cr}, \mathrm{Mn}$, and $\mathrm{Fe}$ and underabundant He. A significant $\mathrm{Ca}$ and $\mathrm{Hg}$ isotopic anomaly has recently been discovered by Castelli \& Hubrig (in preparation) in a UVES spectrum of this star. However, no lines of $\mathrm{Hg}$ or other heavy elements were observed in our sample stars.

A vertical abundance inhomogeneous distribution was found in HgMn and in other subgroups of CP stars. The Ca stratification and isotope separation in several chemically peculiar stars has recently been investigated by Cowley et al. $(2007,2009)$ and by Ryabchikova et al. (2008) who show that heavy Ca isotopes in a few stars are most likely concentrated towards the higher atmospheric layers. No sign of an elemental vertical inhomogeneous distribution was found in any spectrum of the studied stars. In addition, the lines lying in different spectral regions can be reproduced with the same abundance within the limits of the loggf's accuracy.

As shown by Michaud et al. (2008), the abundance anomalies in BHB stars are believed to be caused by atomic diffusion, radiative accelerations leading for instance to the observed $\mathrm{Fe}$ overabundances. The abundance anomalies of $\mathrm{Al}, \mathrm{Si}, \mathrm{S}, \mathrm{Ca}, \mathrm{Ti}$, $\mathrm{Cr}$, and $\mathrm{Ni}$, but not of $\mathrm{Mg}$, are also reasonably well-reproduced by these authors in stellar evolution models, which include all effects of atomic diffusion from the ZAMS to the horizontal branch. Still, it is not clear yet to what extent the different abundances of the two different populations can be explained in terms of the different evolutionary history or of different atmospheric phenomena, such as diffusion. The link with atomic diffusion is strengthened by the observed slow rotation, a feature that is also a characteristic of $\mathrm{HgMn}$ stars and is required to allow the slow diffusion processes to be effective. At least $2 / 3$ of the known $\mathrm{HgMn}$ stars belong to spectroscopic binary systems (Hubrig \& Mathys 1995), and the slow rotation could in principle be explained with binarity, where the components are synchronized with $P_{\text {rot }}=P_{\text {orb}}$. In contrast to $\mathrm{HgMn}$ stars, only very few BHB stars are known to belong to a binary system. Moni Bidin et al. (2006) used the ESO VLT-FORS2 instrument to obtain medium-resolution $(R \approx 4100)$ spectra of 51 hot HB stars with $8000 \mathrm{~K} \leq T_{\text {eff }} \leq 32000 \mathrm{~K}$ during four consecutive nights, but no close binary system has been detected. For both stars, T183 and B2151 observed on two consecutive nights, small radial velocity changes were found indicating that the fraction of binary systems among BHB stars could be larger than estimated in the previous studies.

In other groups of chemically peculiar stars, magnetic fields are believed to be responsible for their slow rotation. The presence of global magnetic fields contributes to suppressing turbulence and leads to different diffusion velocities depending on the 

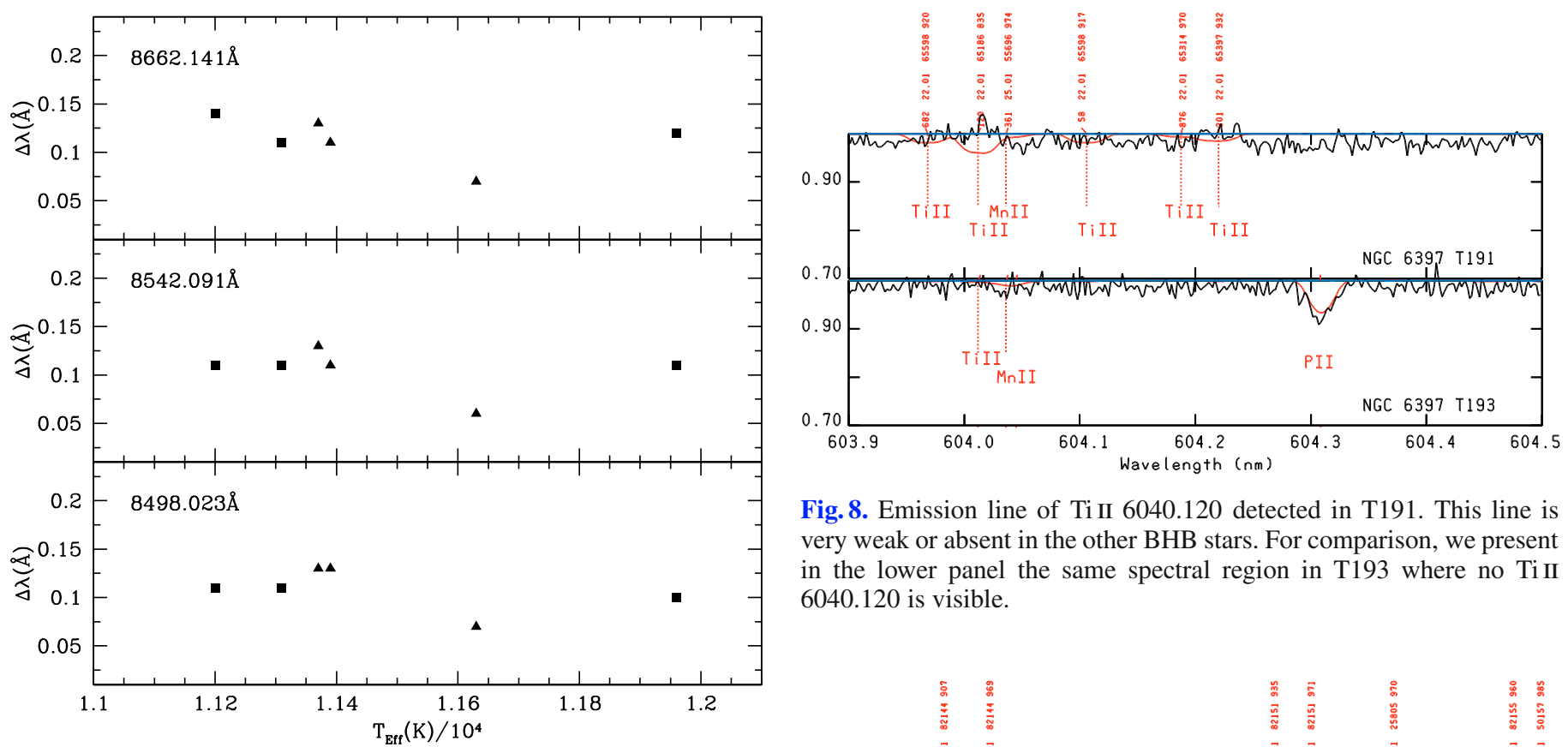

Fig. 8. Emission line of Ti II 6040.120 detected in T191. This line is very weak or absent in the other BHB stars. For comparison, we present in the lower panel the same spectral region in T193 where no Ti II 6040.120 is visible.
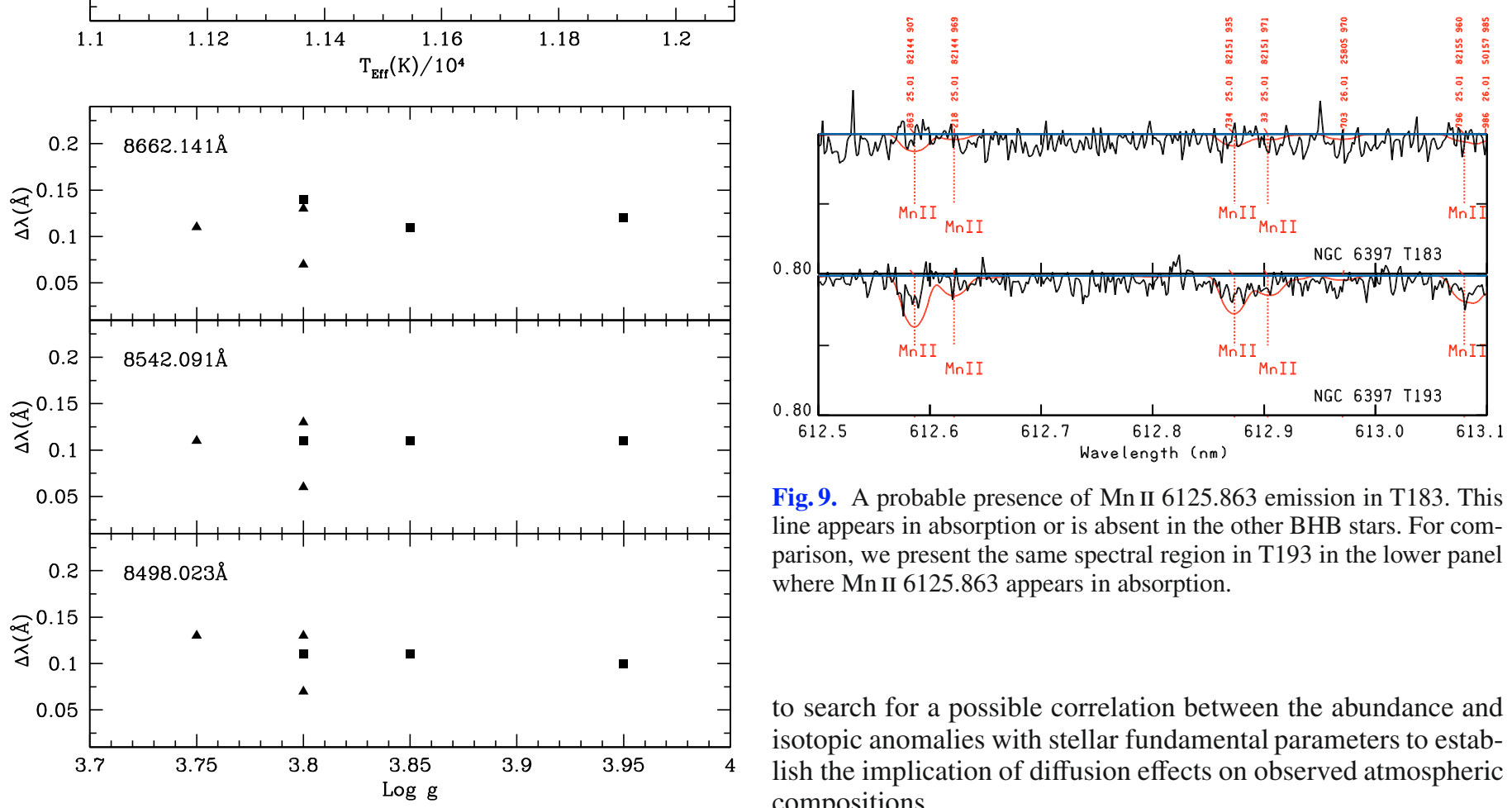

Fig. 7. Ca isotopic shifts for each studied BHB star for Ca II triplet lines $(\lambda 8498, \lambda 8542$, and $\lambda 8662)$ versus $T_{\text {eff }}$ and $\log g$. Squares represent BHB stars in NGC 6397 and triangles represent BHB stars in NGC 6752.

Fig. 9. A probable presence of Mn II 6125.863 emission in T183. This line appears in absorption or is absent in the other BHB stars. For comparison, we present the same spectral region in T193 in the lower panel where Mn II 6125.863 appears in absorption.

to search for a possible correlation between the abundance and isotopic anomalies with stellar fundamental parameters to establish the implication of diffusion effects on observed atmospheric compositions.

Acknowledgements. We would like to thank M. Schöller for his help with the layout of the manuscript and useful suggestions.

\section{Appendix A: Lines used for the abundance analysis}

field inclination and strength. To our knowledge, there has been no attempt so far to detect magnetic fields in BHB stars of globular clusters. Future spectropolarimetric studies to establish the presence or absence of magnetic fields in these stars will provide important clues to their potential role in slowing them down.

Furthermore, it is important to obtain high-resolution, high $\mathrm{S} / \mathrm{N}$ spectra for a larger sample of BHB stars with $T_{\text {eff }}$ values spanning the range from $10000 \mathrm{~K}$ to much higher $T_{\text {eff }}$ than in our sample stars, belonging to various globular clusters with different metallicity. The gradually increasing number of large telescopes equipped with high-resolution spectrographs ensures the availability of such spectroscopic studies. They should be used

Table A.1 lists the lines that were investigated in the spectra of the six stars. The wording "not obs" is given for lines not present in the spectra, while the wordings "profile" and "blend" are given for lines observed well in the spectra but that do not have measurable equivalent widths either because the noise affects the profile too much or because other components affect the line. These wordings also indicate lines for which adequate equivalent widths cannot be computed as in the cases of $\mathrm{Mg}$ II at $4481 \AA$, which is a blend of transitions belonging to the same multiplet, of most Mn II lines which are affected by hyperfine structure, of the Ca II infrared triplet which is a blend of isotopic 
Table A.1. Analysed lines in the stellar spectra and measured equivalent widths in mA.

\begin{tabular}{|c|c|c|c|c|c|c|c|c|c|c|}
\hline Species & $\lambda(\AA)$ & $\log g f$ & Ref. & $E_{i}\left(\mathrm{~cm}^{-1}\right)$ & $W(\mathrm{~T} 183)$ & $W(\mathrm{~T} 191)$ & $W(\mathrm{~T} 193)$ & $W(\mathrm{~B} 0652)$ & $W(\mathrm{~B} 2151)$ & $W(\mathrm{~B} 2206)$ \\
\hline C II & 4267.261 & 0.716 & NIST3 & 145550.700 & not obs & not obs & not obs & not obs & not obs & not obs \\
\hline $\mathrm{N}_{\mathrm{I}}$ & 8680.282 & 0.347 & NIST3 & 83364.620 & not obs & not obs & not obs & not obs & not obs & not obs \\
\hline $\mathrm{N}_{\mathrm{I}}$ & 8683.403 & 0.087 & NIST3 & 88317.830 & not obs & not obs & not obs & not obs & not obs & not obs \\
\hline O I & 7771.944 & 0.369 & NIST3 & 73768.200 & not obs & profile & not obs & not obs & not obs & not obs \\
\hline O I & 7774.166 & 0.223 & NIST3 & 73768.200 & not obs & profile & not obs & not obs & not obs & not obs \\
\hline O I & 7775.388 & 0.002 & NIST3 & 73768.200 & not obs & profile & not obs & not obs & not obs & not obs \\
\hline $\mathrm{NaI}$ & 5889.950 & 0.108 & NIST3 & 0.000 & not obs & not obs & not obs & profile & not obs & profile \\
\hline $\mathrm{NaI}$ & 5895.924 & -0.194 & NIST3 & 0.000 & not obs & not obs & not obs & profile & not obs & profile? \\
\hline Mg II & 4481.126 & 0.749 & NIST3 & 71490.190 & profile & profile & profile & profile & profile & profile \\
\hline Mg II & 4481.150 & -0.553 & NIST3 & 71490.190 & profile & profile & profile & profile & profile & profile \\
\hline Mg II & 4481.325 & 0.594 & NIST3 & 71491.063 & profile & profile & profile & profile & profile & profile \\
\hline $\mathrm{Al} \mathrm{I}$ & 3944.006 & -0.638 & NIST3 & 0.000 & not obs & not obs & not obs & not obs & not obs & not obs \\
\hline $\mathrm{Al}$ I & 3961.520 & -0.336 & NIST3 & 112.061 & not obs & not obs & not obs & not obs & not obs & not obs \\
\hline Si II & 3853.665 & -1.341 & NIST3 & 55309.350 & not obs & profile & not obs & not obs & not obs & not obs \\
\hline Si II & 3856.018 & -0.406 & NIST3 & 55325.180 & profile & profile & not obs & not obs & not obs & not obs \\
\hline Si II & 3862.595 & -0.757 & NIST3 & 55309.350 & not obs & profile & not obs & not obs & not obs & not obs \\
\hline Si II & 4130.872 & -0.783 & NIST3 & 79355.020 & not obs & profile & not obs & not obs & not obs & not obs \\
\hline Si II & 4130.894 & 0.552 & NIST3 & 79355.020 & not obs & profile & not obs & not obs & not obs & not obs \\
\hline P II & 4044.576 & 0.481 & $\mathrm{~K}, \mathrm{MRB}$ & 107360.250 & profile & not obs & profile & 20.9 & profile & profile \\
\hline P II & 4127.559 & -0.110 & $\mathrm{~K}, \mathrm{KP}$ & 103667.860 & profile & not obs & not obs & 10.6 & profile & profile \\
\hline P II & 4420.712 & -0.329 & NIST3 & 88893.220 & profile & not obs & 7.3 & 17.7 & not obs & 5.3 \\
\hline P II & 4475.270 & 0.440 & NIST3 & 105549.670 & profile & not obs & 9.1 & 13.1 & not obs & 7.1 \\
\hline P II & 6024.178 & 0.137 & NIST3 & 86743.960 & profile & not obs & profile & 37.9 & profile & profile \\
\hline P II & 6034.039 & -0.220 & NIST3 & 86597.550 & profile & not obs & profile & 27.4 & profile & profile \\
\hline P II & 6043.084 & 0.416 & NIST3 & 87124.600 & profile & not obs & 18.8 & 42.9 & profile & 12.8 \\
\hline S II & 4153.068 & 0.617 & NIST3 & 128233.200 & not obs & not obs & not obs & not obs & not obs & not obs \\
\hline S II & 4162.665 & 0.777 & NIST3 & 128599.160 & not obs & not obs & not obs & not obs & not obs & not obs \\
\hline Ca II & 3933.663 & 0.135 & NIST3 & 0.000 & profile & profile & profile & profile & profile & profile \\
\hline Ca II & 3968.469 & -0.180 & NIST3 & 0.000 & profile & profile & profile & profile & profile & profile \\
\hline Ca II & 8498.023 & -1.450 & GAL & 13650.190 & profile & profile & profile & profile & profile & profile \\
\hline Ca II & 8542.091 & -0.500 & GAL & 13710.880 & profile & profile & profile & profile & profile & profile \\
\hline Ca II & 8662.141 & -0.760 & GAL & 13650.190 & profile & profile & profile & profile & profile & profile \\
\hline Sc II & 4246.822 & 0.242 & NIST3 & 2540.950 & not obs & not obs & not obs & not obs & not obs & not obs \\
\hline Sc II & 4314.083 & -0.100 & NIST3 & 4987.790 & not obs & not obs & not obs & not obs & not obs & not obs \\
\hline Ti II & 4053.821 & -1.130 & PTP & 15265.620 & 31.8 & profile & 15.3 & profile & 13.6 & 15.0 \\
\hline Ti II & 4161.529 & -2.360 & NIST3 & 8744.250 & profile & 37.8 & profile & not obs & not obs & profile \\
\hline Ti II & 4163.644 & -0.130 & PTP & 20891.660 & 50.1 & 80.6 & 38.1 & 16.7 & 32.3 & 36.0 \\
\hline Ti II & 4287.873 & -1.790 & PTP & 8710.440 & 21.7 & 54.1 & profile & profile & 9.2 & 8.7 \\
\hline Ti II & 4290.215 & -0.850 & РTP & 9395.710 & 53.8 & 93.8 & 36.6 & 13.6 & 34.5 & 34.9 \\
\hline Ti II & 4294.094 & -0.930 & РTP & 9744.250 & 55.7 & 83.0 & 39.3 & blend & blend & 37.3 \\
\hline Ti II & 4300.042 & -0.440 & РТP & 9518.060 & 69.3 & 103.8 & 58.2 & 31.4 & 49.4 & 50.4 \\
\hline Ti II & 4301.922 & -1.150 & РTP & 9363.620 & 39.9 & 70.2 & 26.5 & 7.8 & 21.5 & 21.9 \\
\hline Ti II & 4312.860 & -1.100 & PTP & 9518.060 & 51.4 & 78.9 & 36.3 & blend & 26.2 & 25.8 \\
\hline Ti II & 4314.971 & -1.100 & PTP & 9363.620 & 46.1 & 75.6 & 35.7 & blend & blend & blend \\
\hline Ti II & 4367.652 & -0.860 & PTP & 20891.660 & 28.2 & 59.0 & profile & profile & 10.2 & 13.5 \\
\hline Ti II & 4386.847 & -0.960 & PTP & 20951.620 & 21.4 & 53.5 & 12.4 & not obs & 9.6 & 10.4 \\
\hline Ti II & 4394.059 & -1.780 & РTP & 9850.900 & 16.7 & 48.4 & 8.7 & not obs & 6.4 & 7.1 \\
\hline Ti II & 4395.031 & -0.540 & PTP & 8744.250 & 65.1 & 94.0 & 53.0 & 30.3 & 48.1 & 50.9 \\
\hline Ti II & 4399.765 & -1.190 & PTP & 9975.920 & 37.0 & 66.4 & 24.0 & 11.9 & 20.4 & 22.1 \\
\hline Ti II & 4411.072 & -0.670 & РTP & 24961.030 & 22.2 & 51.6 & 15.3 & not obs & 10.6 & 12.0 \\
\hline Ti II & 4417.714 & -1.190 & РTP & 9395.710 & 37.2 & 70.5 & 25.2 & 11.6 & 22.3 & 22.0 \\
\hline Ti II & 4418.331 & -1.970 & PTP & 9975.920 & 12.3 & 42.1 & profile & not obs & 7.1 & 5.8 \\
\hline Ti II & 4443.801 & -0.720 & РTP & 8710.440 & 65.6 & 90.6 & 46.5 & 24.6 & 42.5 & 44.1 \\
\hline Ti II & 4450.482 & -1.520 & PTP & 8744.250 & 32.0 & 64.9 & profile & blend & 15.4 & 14.1 \\
\hline Ti II & 4464.448 & -1.810 & PTP & 9363.620 & 19.0 & 51.8 & 13.0 & not obs & 6.8 & 8.3 \\
\hline Ti II & 4468.492 & -0.620 & NIST3 & 9118.260 & 64.3 & 90.5 & 48.0 & 23.9 & 44.0 & 45.7 \\
\hline Ti II & 4488.325 & -0.510 & PTP & 25192.710 & 28.3 & 59.1 & 18.8 & profile & 14.9 & 16.0 \\
\hline
\end{tabular}


Table A.1. continued.

\begin{tabular}{|c|c|c|c|c|c|c|c|c|c|c|}
\hline Species & $\lambda(\AA)$ & $\log g f$ & Ref. $^{a}$ & $E_{i}\left(\mathrm{~cm}^{-1}\right)$ & $W(\mathrm{~T} 183)$ & $W(\mathrm{~T} 191)$ & $W(\mathrm{~T} 193)$ & $W(\mathrm{~B} 0652)$ & $W(\mathrm{~B} 2151)$ & $W(\mathrm{~B} 2206)$ \\
\hline Ti II & 4501.270 & -0.770 & PTP & 8997.710 & 72.4 & 94.9 & 45.8 & 26.4 & 44.7 & 47.5 \\
\hline Ti II & 4563.757 & -0.690 & PTP & 9050.900 & 51.9 & 87.7 & 40.0 & 20.2 & 30.8 & 37.3 \\
\hline Ti II & 4571.971 & -0.320 & PTP & 12676.970 & 71.7 & 101.5 & 57.3 & 33.4 & 49.5 & 55.6 \\
\hline Ti II & 4805.085 & -1.120 & NIST3 & 16625.110 & 33.6 & 64.9 & 20.8 & profile & 16.9 & 15.5 \\
\hline Ti II & 4911.195 & -0.610 & PTP & 25192.790 & 25.0 & 59.9 & 17.7 & profile & 12.1 & 12.9 \\
\hline Cr II & 4558.650 & -0.410 & SL & 32854.310 & 27.3 & 48.5 & 47.0 & 30.6 & 32.4 & 36.2 \\
\hline Cr II & 4588.199 & -0.640 & NIST3 & 32836.680 & 22.0 & 37.8 & 40.2 & blend & 26.9 & 31.8 \\
\hline Cr II & 4618.803 & -0.860 & SL & 32854.950 & 12.5 & 32.2 & 27.3 & 13.7 & 18.5 & 22.1 \\
\hline Cr II & 4634.070 & -0.990 & SL & 32844.760 & profile & 26.7 & 23.7 & profile & 13.7 & 16.4 \\
\hline $\mathrm{Mn} \mathrm{II}^{1}$ & 4206.367 & -1.590 & K03Mn & 43528.640 & profile & profile & profile & profile & not obs & profile \\
\hline Mn II & 4242.333 & -1.288 & K03Mn & 49820.160 & profile & profile & blend & blend & blend & blend \\
\hline Mn II & 4252.963 & -1.172 & K03Mn & 49889.860 & profile & profile & profile & profile & profile & profile \\
\hline Mn II & 4253.025 & -2.312 & K03Mn & 43395.380 & profile & profile & profile & profile & not obs & profile \\
\hline Mn II & 4253.112 & -2.079 & $\mathrm{~K} 03 \mathrm{Mn}$ & 49889.860 & profile & profile & profile & profile & not obs & profile \\
\hline Mn II $^{1}$ & 4282.490 & -1.698 & K03Mn & 44521.520 & profile & profile & profile & blend & blend & profile \\
\hline $\mathrm{Fe} \mathrm{I}^{*}$ & 3815.840 & 0.232 & FW06 & 11976.238 & 40.8 & 22.8 & 33.2 & 45.2 & 46.2 & 38.7 \\
\hline $\mathrm{Fe} \mathrm{I}^{*}$ & 3820.425 & 0.119 & FW06 & 6928.268 & 50.4 & 30.3 & 45.2 & 46.3 & 55.8 & 49.4 \\
\hline $\mathrm{Fe} \mathrm{I}^{*}$ & 3856.371 & -1.286 & FW06 & 415.933 & 13.0 & profile & profile & profile & 15.3 & 14.8 \\
\hline $\mathrm{Fe} \mathrm{I}^{*}$ & 3859.911 & -0.710 & FW06 & 0.000 & 27.8 & 21.2 & 27.8 & 31.6 & 36.8 & 33.9 \\
\hline $\mathrm{Fe} \mathrm{I}$ & 4005.242 & -0.610 & FW06 & 12560.933 & profile & profile & 13.3 & 18.9 & 15.8 & 7.6 \\
\hline $\mathrm{Fe} I$ & 4045.812 & 0.280 & FW06 & 11976.238 & 41.9 & 20.1 & 38.1 & 42.9 & 44.1 & 45.1 \\
\hline $\mathrm{Fe} I$ & 4071.738 & -0.022 & FW06 & 12968.553 & 28.5 & profile & 23.5 & 27.8 & 30.1 & 31.9 \\
\hline $\mathrm{Fe} \mathrm{I}$ & 4202.029 & -0.708 & FW06 & 11976.238 & 10.5 & profile & 9.3 & 16.0 & 12.3 & 14.4 \\
\hline $\mathrm{Fe} \mathrm{I}$ & 4219.360 & 0.000 & FW06 & 28819.952 & profile & not obs & profile & not obs & 7.1 & 7.0 \\
\hline $\mathrm{Fe} \mathrm{I}$ & 4235.936 & -0.341 & FW06 & 43163.323 & profile & not obs & profile & profile & 11.1 & 10.7 \\
\hline $\mathrm{Fe} I$ & 4271.760 & -0.164 & FW06 & 11976.238 & 29.4 & profile & 26.9 & 31.9 & 30.5 & 32.2 \\
\hline $\mathrm{Fe} \mathrm{I}$ & 4383.545 & 0.200 & FW06 & 11976.238 & 43.3 & 21.7 & 37.5 & 38.8 & 45.7 & 41.5 \\
\hline $\mathrm{Fe} I$ & 4404.750 & -0.142 & FW06 & 12560.933 & 26.2 & profile & 27.4 & 29.3 & 29.2 & 32.3 \\
\hline $\mathrm{Fe} \mathrm{I}$ & 4415.122 & -0.615 & FW06 & 12968.553 & 17.6 & profile & 14.2 & 18.1 & 16.6 & 17.2 \\
\hline $\mathrm{Fe}$ II & 4122.668 & -3.300 & FW06 & 20830.582 & 39.4 & 20.1 & 36.6 & 47.2 & 39.5 & 37.8 \\
\hline $\mathrm{Fe}$ II & 4128.748 & -3.578 & FW06 & 20830.582 & 24.5 & 13.7 & 23.2 & 33.5 & 25.9 & 27.9 \\
\hline $\mathrm{Fe}$ II & 4178.862 & -2.443 & FW06 & 20830.582 & 59.6 & 46.8 & 56.1 & 70.4 & 61.1 & 66.5 \\
\hline $\mathrm{Fe}$ II & 4258.154 & -3.480 & FW06 & 21812.055 & 31.0 & 14.3 & 27.2: & 38.8 & 31.7 & 29.2 \\
\hline $\mathrm{Fe}$ II & 4273.326 & -3.300 & FW06 & 21812.055 & 32.8 & 18.5 & 30.9 & 43.6 & 33.9 & 34.1 \\
\hline $\mathrm{Fe}$ II & 4296.572 & -2.930 & FW06 & 21812.055 & 50.2 & 28.3 & 42.9 & 58.1 & 46.9 & 47.3 \\
\hline Fe II & 4303.176 & -2.610 & FW06 & 21812.055 & 65.5 & 49.0 & 59.4 & 68.6 & 61.4 & 61.9 \\
\hline $\mathrm{Fe}$ II & 4369.411 & -3.580 & FW06 & 22409.852 & 27.9 & 13.0 & 22.7 & 33.8 & 25.3 & 26.6 \\
\hline Fe II & 4385.387 & -2.580 & FW06 & 22409.852 & 62.7 & 37.5 & 57.0 & 63.6 & 57.7 & 57.3 \\
\hline $\mathrm{Fe}$ II & 4413.601 & -4.190 & FW06 & 21581.638 & 10.0 & not obs & 12.7 & 19.1 & 14.8 & 11.7 \\
\hline $\mathrm{Fe}$ II & 4416.830 & -2.600 & FW06 & 22409.852 & 57.1 & 38.9 & 55.3 & 65.6 & 63.0 & 58.9 \\
\hline $\mathrm{Fe}$ II & 4491.405 & -2.640 & FW06 & 23031.300 & 53.7 & 34.5 & 50.7 & 64.0 & 52.5 & 52.3 \\
\hline Fe II & 4508.288 & -2.350 & FW06 & 23031.300 & 69.1 & 48.9 & 65.2 & 77.4 & 68.6 & 68.1 \\
\hline $\mathrm{Fe}$ II & 4515.339 & -2.360 & FW06 & 22939.358 & 64.8 & 43.8 & 58.1 & 68.8 & 62.7 & 65.1 \\
\hline Fe II & 4520.224 & -2.620 & FW06 & 22637.205 & 55.2 & 40.5 & 52.3 & 63.5 & 57.6 & 56.9 \\
\hline Fe II & 4522.634 & -1.990 & FW06 & 22939.358 & 69.5 & 54.6 & 69.4 & 81.3 & 70.1 & 74.4 \\
\hline Fe II & 4541.524 & -2.970 & FW06 & 23031.300 & 47.0 & 27.3 & 41.5 & 54.0 & 48.3 & 45.7 \\
\hline $\mathrm{Fe}$ II & 4549.192 & -1.705 & K07F & 47674.721 & 39.8 & 21.7 & 36.3 & profile & 37.1 & 37.3 \\
\hline $\mathrm{Fe}$ II & 4549.474 & -1.730 & FW06 & 22810.357 & blend & blend & blend & blend & 82.3: & 81.6: \\
\hline Fe II & 4555.893 & -2.250 & FW06 & 22810.357 & 69.2 & 49.4 & 61.0 & 69.2 & 64.6 & 64.5 \\
\hline $\mathrm{Fe}$ II & 4576.340 & -2.920 & FW06 & 22939.358 & 47.5 & 28.7 & 41.0 & 56.3 & 44.4 & 46.4 \\
\hline Fe II & 4582.835 & -3.062 & FW06 & 22939.358 & 41.7 & 21.6 & 37.7 & 45.6 & 39.2 & 37.4 \\
\hline $\mathrm{Fe}$ II & 4583.837 & -1.740 & FW06 & 22637.205 & 88.9 & 63.8 & 86.2 & 102.3 & 82.1 & 82.1 \\
\hline $\mathrm{Fe} \mathrm{II}^{*}$ & 6147.741 & -2.702 & K07F & 31364.440 & 32.4 & 20.0 & 26.690 & 49.4 & 39.2 & 33.6 \\
\hline Fe II* & 6149.258 & -2.703 & K07F & 31368.450 & 35.0 & bad spect. & 29.8 & 45.5 & 34.1 & 36.7 \\
\hline $\mathrm{Fe} \mathrm{II}^{*}$ & 6383.722 & -2.235 & K07F & 44784.761 & 17.6 & not obs & 14.8 & 25.9 & 19.1 & 19.8 \\
\hline $\mathrm{Fe} \mathrm{II}^{*}$ & 6416.928 & -2.718 & K07F & 31387.948 & 38.0 & 18.1 & 28.4 & 43.7 & 30.8 & 28.1 \\
\hline $\mathrm{Fe} \mathrm{II}^{*}$ & 6456.383 & -2.057 & K07F & 31483.176 & 60.7 & 40.8 & 53.8 & 73.7 & 56.4 & 56.6 \\
\hline
\end{tabular}


Table A.1. continued.

\begin{tabular}{|c|c|c|c|c|c|c|c|c|c|c|}
\hline Species & $\lambda(\AA)$ & $\log g f$ & Ref. $^{a}$ & $E_{i}\left(\mathrm{~cm}^{-1}\right)$ & $W(\mathrm{~T} 183)$ & $W(\mathrm{~T} 191)$ & $W(\mathrm{~T} 193)$ & $W(\mathrm{~B} 0652)$ & $W(\mathrm{~B} 2151)$ & $W(\mathrm{~B} 2206)$ \\
\hline Co II & 4160.657 & -1.751 & K06 & 27484.371 & not obs & profile & not obs & not obs & not obs & not obs \\
\hline Ni II & 4015.474 & -2.410 & $\mathrm{~K} 03 \mathrm{Ni}$ & 32523.540 & profile & profile & profile & profile & not obs & profile \\
\hline Ni II & 4067.031 & -1.834 & $\mathrm{~K} 03 \mathrm{Ni}$ & 32499.530 & profile & profile & profile & profile & profile & profile \\
\hline Ga II & 4255.722 & 0.634 & RS94 & 113842.190 & not obs & not obs & not obs & not obs & not obs & not obs \\
\hline Ga II & 6334.069 & 1.000 & RS94 & 102944.550 & not obs & not obs & not obs & not obs & not obs & not obs \\
\hline Sr II & 4077.709 & 0.151 & NIST3 & 0.000 & not obs & not obs & not obs & not obs & not obs & not obs \\
\hline Y II & 3950.349 & -0.490 & $\mathrm{~K}, \mathrm{HL}$ & 840.213 & profile & profile & 12.6 & 25.1 & profile & profile \\
\hline Y II & 4883.682 & 0.070 & $\mathrm{~K}, \mathrm{HL}$ & 8743.316 & profile & profile & 17.2 & 21.0 & profile & 11.9 \\
\hline Y II & 4900.120 & -0.090 & $\mathrm{~K}, \mathrm{HL}$ & 8328.041 & profile & profile & 12.3 & 24.6 & profile & 12.3 \\
\hline Zr II & 4149.198 & -0.040 & LNAJ & 6467.610 & not obs & not obs & not obs & not obs & not obs & profile \\
\hline Xe II & 4603.005 & 0.017 & NIST3 & 95064.380 & not obs & not obs & not obs & not obs & not obs & not obs \\
\hline Xe II & 4844.330 & 0.491 & NIST3 & 93068.440 & not obs & not obs & not obs & not obs & not obs & not obs \\
\hline Ba II & 4554.029 & -0.161 & NIST3 & 0.000 & not obs & not obs & not obs & not obs & not obs & not obs \\
\hline Ba II & 4934.076 & -0.458 & NIST3 & 0.000 & not obs & not obs & not obs & not obs & not obs & not obs \\
\hline Pt II & 4514.124 & -1.480 & DSJ & 29261.970 & not obs & not obs & not obs & not obs & not obs & not obs \\
\hline Au II & 4016.067 & -1.880 & RW & 48510.890 & not obs & not obs & not obs & not obs & not obs & not obs \\
\hline $\mathrm{Au}$ II & 4052.790 & -1.690 & RW & 48510.890 & not obs & not obs & not obs & not obs & not obs & not obs \\
\hline $\mathrm{Hg}$ II & 3983.890 & -1.510 & NIST3 & 35514.000 & not obs & not obs & not obs & not obs & not obs & not obs \\
\hline
\end{tabular}

Note: He I is not included. It is discussed in the text. The Fe I and Fe II lines marked with an asterisk were used only to estimate the error on $T_{\text {eff }}$ due to the particular sample of lines (see Sect. 3.1).

1 The hyperfine structure was considered in the line profile computations. ${ }^{a}$ (NIST3) NIST Atomic Spectra Database, version 3 at http://physics.nist.gov; (DSJ) Dworetsky et al. (1984); (FW06) Fuhr \& Wiese (2006); (GAL) Gallager (1967); (LNAJ) Ljung et al. (2006); (PTP) Pickerin et al. (2002); (RS94) Ryabchikova \& Smirnov (1994); (RW) Rosberg \& Wyart (1997); (SL) Sigut \& Landstreet (1990); K03Mn: http://kurucz . harvard. edu/atoms/2501/gf2501.pos; K03Ni: http://kurucz.harvard.edu/atoms/2801/gf2801. pos; K06: http://kurucz.harvard.edu/atoms/2701/gf2701.pos; K07F: http://kurucz.harvard.edu/atoms/2601/gf2601.pos. For these lines the Kurucz $\log g f$ 's give a better agreement with the observations than the FW06 $\log g f^{\prime} s$; "K" before another log $g f$ source means that the $\log g f$ is from Kurucz files available at http://kurucz.harvard.edu/linelists/gf100/; (HL) Hannaford et al. (1982); (KP) Kurucz\& Peytremann (1975); (MRB) Miller et al. (1971).

components, and so on. For the remaining lines the measured equivelent widths are presented in the table.

\section{References}

Anders, E., \& Grevesse, N. 1989, Geochim. Cosmochim. Acta, 53, 197 Anthony-Twarog, B. J., \& Twarog, B. A. 2000, AJ, 120, 3111 Ballester, P., Grosbol, P., Banse, K., et al. 2000, Proc. SPIE, 4010, 246 Behr, B. B. 2003, ApJS, 149, 67

Behr, B. B., Cohen, J. G., \& McCarthy, J. K. 2000, ApJ, 531, L37

Bessell, M. S., Castelli, F., \& Plez, B. 1998, A\&A, 333, 231

Bonifacio, P., Castelli, F., \& Hack M. 1995, A\&AS, 110, 441

Buonanno, R., Caloi, V., Castellani, V., et al. 1986, A\&AS, 66, 79

Castelli, F. 2005a, MSAIS, 8, 25

Castelli, F. 2005b, MSAIS, 8, 44

Castelli, F., \& Hubrig, S. 2004a, A\&A, 421, L1

Castelli F., \& Hubrig, S. 2004b, A\&A, 425, 263

Cowley, C. R., \& Hubrig, S. 2005, A\&A, 432, L21

Cowley, C. R., Hubrig, S., Castelli, F., et al. 2007, MNRAS, 377, 1579

Cowley, C. R., Hubrig, S., \& González, J. F. 2009, [arXiv: 0903. 0611]

de Boer, K. S., Schmidt, J. H. K., \& Heber, U. 1995, A\&A, 303, 95

Dolk, L., Wahlgren, G. M., \& Hubrig, S. 2003, A\&A, 402, 299

Dworetsky, M. M., Storey, P. J., \& Jacobs, J. M. 1984, Phys. Scr., 8, 39

Fabbian, D., Recio-Blanco, A., Gratton, R. G., \& Piotto, G. 2005, A\&A, 434, 235

Fuhr, J. R., \& Wiese, W. L. 2006, J. Phys. Chem. Ref. Data, 35, 4

Gallagher, A. 1967, Phys. Rev., 157, 24

Glaspey, J. W., Michaud, G., Moffat, A. F. J., \& Demers, S. 1989, ApJ, 339, 926

Gonzalez, J.-F., Artru, M.-C., \& Michaud, G. 1995, A\&A, 302, 788

Gratton, R. G., Bragaglia, A., Carretta, E., et al. 2003, A\&A, 408, 529

Greenstein, G. S., Truran, J. W., \& Cameron, A. G. W. 1967, Nature, 213, 871

Grevesse, N., \& Sauval, A. J. 1998, Space Sci. Rev., 85, 161

Grundahl, F., Catelan, M., Landsman, W. B., et al. 1999, ApJ, 524, 242

Hannaford, P., Lowe, R. M., Grevesse, N., Biemont, E., \& Whaling, W. 1982, ApJ, 261, 736
Hartoog, M. R. 1979, ApJ, 231, 161

Hoyle, F., \& Schwarzschild, M. 1995, ApJS, 2, 1

Hubrig, S., \& Mathys, G. 1995, Comm. Astroph., 18, 167

Hubrig, S., Castelli, F., \& Mathys, G. 1999, A\&A, 341, 190

Kurucz, R. L. 1993, ATLAS9 Stellar Atmosphere Programs and 2 km/s grid, Kurucz CD-ROM No. 13, Cambridge, Mass.: Smithsonian Astrophysical Observatory

Kurucz, R. L. 2005, MSAIS, 8, 14

Kurucz, R. L., \& Peytremann, E. 1975, SAO Special Rep., 362

Ljung, G., Nilsson, H., Asplund, M., \& Johansson, S. 2006, A\&A, 456, 1181

Martensson-Pendril, A.-M., Ynnerman, A., Wartson, H., et al. 1992, Phys. Rev. A, 45,4675

Michaud, G., Richer, J., \& Richard, O. 2008, ApJ, 675, 1223

Miller, M. H., Roig, R. A., \& Bengtson, R. D. 1971, Phys. Rev. A, 4, 1709

Moehler, S., Sweigart, A. N., Landsman, W. B., et al. 1999, A\&A, 346, L1

Moehler, S., Sweigart, A. V., Landsman, W. B., et al. 2000, A\&A, 360, 120

Momany, Y., Piotto, G., Recio-Blanco, A., et al. 2002, ApJ, 576, L65

Momany, Y., Cassisi, S., Piotto, G., et al. 2003, A\&A, 407, 303

Momany, Y., Bedin, L. R., Cassisi, S., et al. 2004, A\&A, 420, 605

Moni Bidin, C., Moehler, S., Piotto, G., et al. 2006, A\&A, 451, 499

Nörtershäuser, W., Blaum, K., Icker, P., et al. 1998, Eur. Phys. J. D, 2, 33

Pickering, J. C., Thorne, A. P., \& Perez, R. 2002, ApJS, 138, 247

Proffitt, C. R., Brage, T., Leckrone, D. S., et al. 1999, ApJ, 512, 942

Pietrinferni, A., Cassisi, S., Salaris, M., \& Castelli, F. 2006, ApJ, 642, 797

Recio-Blanco, A., Piotto, G., Aparicio, A., \& Renzini, A. 2002, ApJ, 572, L71

Rosberg, M., \& Wyart, J.-F. 1997, Phys. Scr, 55, 690

Ryabchikova, T. A., \& Smirnov, Y. M. 1994, Astron. Rep., 38, 70

Ryabchikova, T. A., Kochukhov, O., \& Bagnulo, S., 2008, A\&A, 480, 811

Sargent, A. W. L. W., \& Jugaku, J. 1961, ApJ, 134, 777

Sbordone, L., Bonifacio, P., Castelli, F., \& Kurucz, R. L. 2004, MSAIS, 5, 93

Sigut, T. A. A. 2001, A\&A, 377, 27

Sigut, T. A. A., \& Landstreet, J. D. 1990, MNRAS, 247, 611

Sosin, C., Dorman, B., Gjorgovski, S. G., et al. 1997, ApJ, 480, L35

Trundle, C., Dufton, P. L., Rolleston, W. R. J., et al. 2001, MNRAS, 328, 291

Wahlgren, G. M., \& Hubrig, S. 2000, A\&A, 362, 13

Wahlgren, G. M., \& Hubrig, S. 2004, A\&A, 418, 1073

Zinn, R. 1985, ApJ, 293, 424 